\title{
A New Multiple-Symbol Differential Detection Strategy for Error-Floor Elimination of IEEE 802.15.4 BPSK Receivers Impaired by Carrier Frequency Offset
}

\author{
Gaoyuan Zhang $\mathbb{D D}^{1,2,3}$ Congzheng Han ${ }^{\mathbb{D}},{ }^{2}$ Baofeng Ji, ${ }^{1,2}$ Congyu Shi ${ }^{D},{ }^{1}$ Ping Xie $\mathbb{D}^{1},{ }^{1}$ \\ and Ling Yang ${ }^{4}$ \\ ${ }^{1}$ School of Information Engineering, Henan University of Science and Technology, Luoyang, China \\ ${ }^{2}$ Key Laboratory of Middle Atmosphere and Global Environment Observation, Institute of Atmospheric Physics, \\ Chinese Academy of Sciences, Beijing, China \\ ${ }^{3}$ National Key Laboratory of Science and Technology on Communications, University of Electronic Science and \\ Technology of China, Chengdu, China \\ ${ }^{4}$ Independent Researcher, Xi'an, China
}

Correspondence should be addressed to Gaoyuan Zhang; zhanggaoyuan407@163.com and Congzheng Han; c.han@mail.iap.ac.cn

Received 22 July 2019; Revised 10 October 2019; Accepted 22 October 2019; Published 26 November 2019

Academic Editor: Xianfu Lei

Copyright (c) 2019 Gaoyuan Zhang et al. This is an open access article distributed under the Creative Commons Attribution License, which permits unrestricted use, distribution, and reproduction in any medium, provided the original work is properly cited.

\begin{abstract}
In this paper, we pay our attention towards the noncoherent demodulation aspect of binary phase shift keying (BPSK) receivers for IEEE 802.15.4 wireless sensor networks (WSNs), and a carrier frequency offset invariant as well as error-floor free multiple-symbol differential detection (MSDD) strategy is proposed over the flat fading channel. This detector is an alternative to the multiplesymbol detector that has been considered almost exclusively in the past. In this new configuration, the receivers do not perform chip-level precompensation as in conventional scheme but bit-level postcompensation. That is, the bit-level autocorrelation operation is first implemented with the "raw" chip sample, and then the carrier frequency offset effect (CFOE) embedded in the achieved statistic is compensated. Correspondingly, the cumulative error in the detection metric is decreased so much that the pervasive error floor for the conventional MSDD scheme is suppressed. Also, complexity efficient estimators for the MSDD scheme are reinvestigated, analyzed, and summarized. Simulation results demonstrate that this new detection strategy may achieve rather more encouraging gain from differential and spread spectrum coding than the conventional single differential coherent detection (SDCD) scheme. The pervasive error floor is also eliminated as compared with conventional MSDD scheme even if the most simple estimator is configured under large bit observation length. Then, much transmitting energy may be saved for each chip symbol, which is practically desired for transmit-only nodes in WSNs.
\end{abstract}

\section{Introduction}

Recent years have witnessed much concentration on pervasive wireless sensor networks (WSNs), especially because of its wide application potentiality in the future "Internet of Things (IoT)" for the "edge access" [1-5]. In particular, more attention is attracted on WSNs with transmit-only nodes. The typical application includes intravehicular sensor network, industrial automation, wireless body area networks (WBANs), telemetry in precision agriculture, and household activities inference [1]. Simply reporting sensed data to the sink reliably and periodically within one hop is now the ultimate and critical goal of dense distributed sensor nodes. Namely, star WSN topologies are necessary to be implemented, and any external control is not needed in this context. For these end-point nodes, it is not necessarily equipped with the high-energy-consuming receiver module [1]. The function-reduced transmit-only nodes can not only achieve improved energy efficiency but also provide excellent support for these applications $[6,7]$.

The IEEE 802.15.4 binary phase shift keying (BPSK) physical layer (PHY) has found appropriate and wide range of application in WSNs $[8,9]$. Previous works pay much attention towards developing single differential coherent 
detection (SDCD) strategy under the condition that the receiver is impaired by carrier frequency offset (CFO) [10-12]. The reader can refer to [12] for a detailed discussion.

Motivated by the aforementioned observations and also to achieve much more performance improvement from differential encoding and then energy saving for the transmit-only node $[13,14]$, in this work, we restrict our attention towards developing a multiple-symbol differential detection (MSDD) scheme for the receiver in the sink node. A preliminary work and result for an initial idea on this issue were presented in [15]. Unfortunately, only the pure additive white Gaussian noise (AWGN) channel is considered in [15]. Furthermore, when we equip the receiver in the sink node with a lowcomplexity estimator, undesired error floor is observed especially for large bit observation length. This follows from the fact that the decision metric is achieved by summation, and the error in each term will be accumulated. Moreover, the number of the summation terms grows rapidly with the observation length $N$. Therefore, we further turn all our attention towards the MSDD scheme without error floor under both pure AWGN and fading channels in the presence of CFO. It should be mentioned that $\mathrm{CFO}$ and spread spectrum coding are not considered in these pioneering works $[12,13]$. For a detailed discussion and an extensive list of references of MSDD, the authors suggest $[13,15]$.

Apparently, the MSDD scheme is much more complex than SDCD. However, it is obviously feasible and essential for both present and future WSNs. Five important reasons are the following:

(i) As shown in [16], the sink node may be integrated into the higher-level system, and thus has less limited energy resources. Motivated by this observation, an augmented receiver for coded offset quadrature phase shift keying (O-QPSK) was introduced with the aid of iterative decoding in [16]. The performance gain is significant because of the combination of encoding, interleaving, and iterative decoding. Especially in future IoT, much computing resource will move towards the edge network to supplement the traditional remote cloud center [17-19]. Increasing computational power in the sink node continues to open the door to more sophisticated algorithms. Then, it will be completely both implementation feasible and energy unlimited for a more complex noncoherent receiver in the sink node.

(ii) Although the MSDD scheme is time consuming, the typical refresh rate for some critical WSN applications such as condition is mandated in the order of minutes or hours [20]. Thus, the MSDD scheme is particularly well suited to these delay-tolerant but performance-sensitive WSNs, which have quite relaxed requirement on latency.

(iii) Without sacrificing the quality of service (QoS), further reduction in energy that is distributed for each chip symbol may be achieved in the transmitonly terminal node. The reason behind this is the fact that our proposed augmented detector can yield rather encouraging reduced packet-error rate (PER). Therefore, it is clearly desirable in terminal devices for energy saving and then maximum service life of WSNs.

(iv) Our proposed augmented detector outperforms the detection schemes for uncoded O-QPSK given in [21-24]. Then, it is particularly well suited to latency-tolerant but error-intolerant applications, such as remote sensing of environment, farming monitoring systems, and smart power meters.

(v) Our proposed MSDD scheme may also exhibit its advantage in standard WSN systems with fullfledged transceivers. Because of giving feedback in the automatic repeat request (ARQ) mechanism [8], the traditional detection scheme can be classified as the close-loop technique [25]. However, no feedback may be required owing to further reliability improvement of our MSDD scheme [26], which can be classified as the open-loop technique [25]. In this context, the transceivers in both ends benefit from the less energy that will be consumed by retransmissions, and further energy can be saved by not sending and listening to automatic acknowledgements at all.

In this work, the main contribution is that a simple and robust MSDD scheme without error floor is improved for BPSK receivers in sink nodes of WSNs. Especially, we are extending and modifying the previous work [15] mainly in four aspects as follows:

(i) We have assumed in [15] that the desired signal was constant in amplitude and not subject to fading due to multipath propagation or blockage. We now examine this constraint, and the detection is clarified in more detail under fading together with AWGN channels.

(ii) In [15], we turn our attention towards the performance and implementation of an efficient estimator to lowering the error floor of the detection scheme. However, in this work, a new detection strategy to be compatible with the estimation scheme is proposed. In this new case, no error floor is exhibited even if the most simple estimator is configured in the receiver and the bit observation length is increased to 8. Note, however, that serious error floor is observed in [15] when the most simple estimator is implemented and the observation length is set to be 4 .

(iii) We develop a more general estimation scheme for CFO effect (CFOE) under slow fading channel. The estimator developed in [15] can be easily integrated into this configuration.

(iv) We evaluate and analyze the detection performance extensively with further simulation study. To show the energy-efficient characteristic of the proposed algorithm to the transmit-only node, we also 
analyze the transmission energy consumption in a real sensor node platform, namely, the Atmel AT86RF212B [27]. Finally, to verify the robustness of the detection algorithm to carrier phase offset (CPO), the performance evaluation result of the proposed MSDD scheme in the presence of random changing CPO is also investigated with simulation.

It is pointed out that we pay all our attention towards uncoded binary differential phase shift keying (DPSK) modulation. The extension to the coded case is straightforward but not pursued here. Moreover, the ensuing analysis is not tailored uniquely for WSN, and thus the results of this paper may be easily extended to include any binary DPSK communication system impaired by both CPO and CFO. Finally, the proposed MSDD scheme may also be easily tailored for uncoded or coded $M$-ary DPSK receivers. Such analyses, however, will be under investigation in our future work.

The remainder of this work takes the following structure. The signal model over the fading channel is given in Section 2. In Section 3, we propose a new strategy to remove the error floor of the conventional multiple-symbol noncoherent detection scheme given in [15] under fading channel. In Section 4, we summarize some estimators, which can be easily developed from conventional tailored schemes for SDCD. In Section 5, we propose a more general estimation scheme under slow fading channel. Section 6 concentrates on the numerical results, while finally Section 7 offers some conclusions and future works.

\section{System Model}

Although we have described similar system models over the AWGN channel in reference [15], for the reader's convenience and also integrity of this work we will repeat it here. Assuming no intersymbol interference and perfect knowledge of timing synchronisation at the receiver over a fading channel, for the $m$ th bit $E_{m}$, we model the complex, baseband, received chip sequence as follows:

$$
r_{m, k}=h_{m, k} s_{m, k} e^{j\left(\omega_{m, k} k T_{c}+\theta_{m, k}\right)}+\eta_{m, k}, \quad 1 \leq k \leq K .
$$

Here, the multiplicative fading is expressed as $h_{m, k}$. The $k$-th bipolar BPSK modulated chip in the $m$-th bit interval is represented as $s_{m, k} \cdot \omega_{m, k}=2 \pi f_{m, k}$ and $\theta_{m, k}$ are arbitrary modulo- $2 \pi$-reduced CFO and CPO in radians, respectively. $T_{c}$ is the chip duration, $\eta_{m, k}$ is a discrete-time, circularly symmetric, zero-mean complex AWGN, and $K=15$ is the length of the pseudorandom number $(\mathrm{PN})$ sequence [8].

Note that the normalized complex Gaussian process $h_{m, k}$ implies a Rayleigh distribution for $\left|h_{m, k}\right|$ when the mean $\overline{h_{m, k}}=0$. In addition, $\left|h_{m, k}\right|$ represents a Rician distribution when the mean $\overline{h_{m, k}} \neq 0$. For simplicity and clarity in describing both estimations and detection schemes, a slow fading channel is considered. In particular, we focus on the case where $h_{m, k}=h, f_{m, k}=f$, and $\theta_{m, k}=\theta$ across a packet transmission. All nuisance parameters are assumed to be unknown for the receivers in this paper unless otherwise specified.

\section{Eliminating the Error Floor of Conventional MSDD by Postcompensation}

As shown in [15], in conventional MSDD algorithm, CFOE is marginalized out with chip-level compensation with an external estimator prior to the bit-level autocorrelation operation. This external estimator is activated only once before each packet detection, and then it can provide an initial estimate of the unknown CFOE. Using this estimate as if it is the true value, the standard perfect-CFO detection scheme given in $[28,29]$ is implemented. Therefore, in this paper, we name conventional MSDD algorithm in [15] as "a chip-level precompensation-based strategy". In order to reduce the detection error floor, an estimator with error to be small enough must be utilized. Consequently, in [15], a new estimation scheme is also proposed in view of the fact that all of the conventional estimation schemes are not compatible. In other words, conventional chip-level compensation-based detection algorithm is an estimator-selective scheme, and the external estimator must be as reliable as possible. Otherwise, the terrible error floor is unavoidable. In the sequel, we will give "a bit-level postcompensation-" based detection strategy, which is error-floor free as well as estimator-nonselective.

The realization that the effect of the CFO can also be wiped out posterior to the bit-level autocorrelation operation is the starting point of our new strategy. That is, we note that the data detection and CFOE compensation process can be swapped. The advantages of this configuration may be twofold. First, in the conventional scheme, the residual estimation error embedded in "each precompensated" chip sample will "accumulate" in each term of equation (6) in [15] by means of the $L_{3}$-term summation operation. The simple idea of our configuration to overcome or weaken this limitation is that we "wholly postcompensate" the CFOE after the bit-level autocorrelation operation. Second, the aforementioned accumulated error will be seriously "amplified" in terms of summation operation as shown in equation (5) of [15], especially when the bit observation length is large. That is, the ultimate error in the detection metric will be improved as the bit observation length increases, and the irreducible error floor may be exhibited. Yet, this limitation is not observed in our new configuration. All of these qualitative observations will be supported by the quantitative results in Section 6. The main implementation process of this new scheme is given in the sequel.

First, we process the "raw" chip sample to obtain a statistic $A_{i, i^{\prime}}$ as follows:

$$
A_{i, i^{\prime}}=\sum_{k=1}^{L_{1}} r_{m-i, k} r_{m-i^{\prime}, k}^{*},
$$

where $L_{1}$ represents the sample number. $i$ and $i^{\prime}$ are the delayed number of the bit interval, and the superscript ${ }^{*}$ is the complex conjugate. Then, reverse modulation is performed after the aforementioned bit-level autocorrelation operation to obtain

$$
B_{i, i^{\prime}}=A_{i, i} e^{-\widehat{j \varphi}_{i^{\prime}-i}}
$$




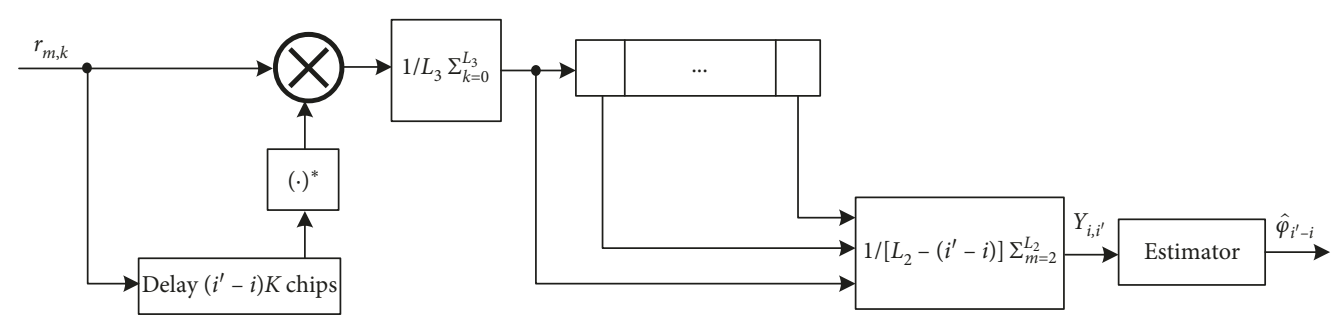

Figure 1: The implementation structure of $\widehat{\varphi}_{i^{\prime}-i}$.

where the estimated CFOE $\hat{\varphi}_{i^{\prime}-i}$ is expressed as

$$
\widehat{\varphi}_{i^{\prime}-i}=\overline{\left(i^{\prime}-i\right) K \omega T_{c}}=g\left(Y_{i^{\prime}, i}\right) \text {. }
$$

Here, the measurement $Y_{i, i^{\prime}}$ is given by

$$
\begin{aligned}
Y_{i, i^{\prime}} & =\frac{1}{\left[L_{2}-\left(i^{\prime}-i\right)\right] L_{3}} \sum_{m=1+i^{\prime}-i}^{L_{2}} \sum_{k=1}^{L_{3}} r_{m, k} r_{m-\left(i^{\prime}-i\right), k}^{*} \\
& =|h|^{2} e^{j\left(i^{\prime}-i\right) K \omega T_{c}}+\eta\left(i^{\prime}-i\right) .
\end{aligned}
$$

Here, $L_{2}$ is the preamble bit observation length, and $2 \leq L_{2} \leq 32$ [8]. $L_{3}$ is the autocorrelation sample number, $1 \leq L_{3} \leq K$, and $\eta\left(i^{\prime}-i\right)$ represents an integrated noise term. Note that although $g\left(Y_{i^{\prime}, i}\right)$ is purposely left generic here, its design is one of the important objectives of the paper. The computation of this estimator will be discussed in Sections 4 and 5. The implementation structure of $\widehat{\varphi}_{i^{\prime}-i}$ is given in Figure 1. For $i=i^{\prime}, \widehat{\varphi}_{i, i^{\prime}}=0$, and then we have, $B_{i, i^{\prime}}=A_{i, i^{\prime}}$.

With the postcompensated and thus quality-improved statistic in (3), the deterministic decision rule is now selecting an absolute phase sequence $\left(\widehat{\phi}_{m-N+1}, \ldots, \widehat{\phi}_{m-1}, \widehat{\phi}_{m}\right)$, which maximizes the complex represented metric $[30,31]$.

$$
\Xi \triangleq \sum_{i=0}^{N} \sum_{i^{\prime}=0}^{N} B_{i, i^{\prime}} e^{-j \Delta \widehat{\phi}_{i, i^{\prime}},}
$$

where $\Delta \widehat{\phi}_{i, i^{\prime}}$ is the relative phase between bit intervals $m-i$ and $m-i^{\prime}$. It is given by

$$
\Delta \widehat{\phi}_{i, i^{\prime}}= \begin{cases}\sum_{i^{\prime \prime}=0}^{i^{\prime}-i-1} \widehat{\phi}_{m-i-i^{\prime \prime}}, & \text { if } i^{\prime}>i, \\ 0, & \text { if } i^{\prime}=i, \\ \sum_{i^{\prime \prime}=0}^{-1} \widehat{\phi}_{m-i-i^{\prime \prime}}, & \text { if } i^{\prime}<i .\end{cases}
$$

The initial but high-complexity metric in (6) can also be written as

$$
\begin{aligned}
\Xi & =\sum_{i=0}^{N-1} B_{i, i^{\prime}}+\sum_{i=0}^{N-1} \sum_{i^{\prime}=i+1, i^{\prime} \neq 1}^{N} \operatorname{Re}\left(B_{i, i^{\prime}} e^{-j \Delta \widehat{\phi}_{i, i^{\prime}}}\right) \\
& =\sum_{i=0}^{N-1} B_{i, i^{\prime}}+2 \sum_{i=0}^{N-1} \sum_{i^{\prime}=i+1}^{N} \operatorname{Re}\left(B_{i, i^{\prime}} e^{-j \sum_{i^{\prime \prime}=0}^{i^{\prime}-i-1} \widehat{\phi}_{m-i-i^{\prime \prime}}}\right) .
\end{aligned}
$$

The first term in (8) may be eliminated because of the independence of the data sequence. Then, we can get an equivalent but low-complexity metric as follows:

$$
\Xi=\sum_{i=0}^{N-1} \sum_{i^{\prime}=i+1}^{N} \operatorname{Re}\left(B_{i, i^{\prime}} e^{-j \sum_{i^{\prime \prime}=0}^{i^{\prime}-i-1} \widehat{\phi}_{m-i-i^{\prime \prime}}}\right) .
$$

The detailed process of our new detection scheme is summarized in Algorithm 1. The conventional MSDD algorithm is summarized as Algorithm 2 for comparison. The implementation structure of new MSDD for $N=4$ is given in Figure 2. For ease of comparison, the structure of conventional MSDD for $N=4$ is given in Figure 3 [15].

\section{Some Evaluated Estimation Schemes}

Before launching a compatible estimation approach for our proposed new MSDD, it is instructive to point out previous estimators to classical SDCD because the estimator for the SDCD scheme over AWGN channel can be easily developed for the proposed MSDD scheme under slow fading channel.

From (5), we can easily achieve a full but computationally intensive estimator as follows: 
Input: $\left\{r_{m, k}\right\}$ : complex baseband chip sample sequence;

$L_{1}$ : sample number for each bit of the actual data;

$L_{2}$ : observation length of the preamble in bit intervals;

$L_{3}$ : number of the autocorrelation sample for $m$-th bit of the preamble;

$K$ : length of the PN sequence;

$L$ : payload length of the PPDU;

$N$ : block length.

Output: $\left(\widehat{\phi}_{m-N+1}, \ldots, \widehat{\phi}_{m-1}, \widehat{\phi}_{m}\right)$ : detection of the actual information phase sequence.

(1) initial $\left\{Y_{0, i}\right\}_{i=1}^{N}=0$, and $K=15$

(2) for $i=1 ; i \leq N ; i++$ do

(3) for $m=1+i ; m \leq L_{2} ; m++$ do

(4) for $k=1 ; k \leq L_{3} ; k++$ do

(5) $\quad Y_{0, i} \longleftarrow Y_{0, i}+r_{m, k} r_{m-i, k}^{*}$

(6) end for

(7) end for

(8) end for

(9) for $i=1 ; i \leq N ; i++$ do

(10) $\quad \widehat{\varphi}_{i} \longleftarrow g\left(Y_{0, i}\right)$;

(11) end for

(12) for $i=m ; i \geq m-N+1 ; i--$ do

(13) for $j=i-1 ; j \geq m-N ; j--$ do

(14) for $k=1 ; k \leq L_{1} ; k++$ do

(15) $\quad A_{m-i, m-j} \longleftarrow A_{m-i, m-j}+r_{i, k} r_{j, k}^{*}$

(16) end for

(17) $\quad B_{m-i, m-j} \longleftarrow A_{m-i, m-j} e^{-\widehat{\varphi_{i-j}}}$

(18) end for

(19) end for

(20) compute the metric $\Xi$ for each hypothetical phase sequence;

(21) find an absolute phase sequence $\left(\widehat{\phi}_{m-N+1}, \ldots, \widehat{\phi}_{m-1}, \widehat{\phi}_{m}\right)$ that maximizes $\Xi$;

(22) return $\left(\widehat{\phi}_{m-N+1}, \ldots, \widehat{\phi}_{m-1}, \widehat{\phi}_{m}\right)$

Algorithm 1: Framework of the proposed MSDD algorithm.

$$
g\left(Y_{i, i^{\prime}}\right)= \begin{cases}\left.\frac{\pi}{2}, \frac{\operatorname{Im}\left(Y_{i, i^{\prime}}\right)}{\operatorname{Re}\left(Y_{i, i^{\prime}}\right)}\right], & \text { if } \operatorname{Re}\left(Y_{i, i^{\prime}}\right)>0 \text { and }\left|\operatorname{Re}\left(Y_{i, i^{\prime}}\right)\right| \geq\left|\operatorname{Im}\left(Y_{i, i^{\prime}}\right)\right|, \\ -\pi+\tan ^{-1}\left[\frac{\operatorname{Im}\left(Y_{i, i^{\prime}}\right)}{\operatorname{Re}\left(Y_{i, i^{\prime}}\right)}\right], & \text { if } \operatorname{Im}\left(Y_{i, i^{\prime}}\right)>0 \text { and }\left|\operatorname{Re}\left(Y_{i, i^{\prime}}\right)\right|<\left|\operatorname{Im}\left(Y_{i, i^{\prime}}\right)\right|, \\ -\frac{\pi}{2}, & \text { if } \operatorname{Im}\left(Y_{i, i^{\prime}}\right)<0 \text { and }\left|\operatorname{Re}\left(Y_{i, i^{\prime}}\right)\right| \geq\left|\operatorname{Im}\left(Y_{i, i^{\prime}}\right)\right|,\end{cases}
$$

Here, $\operatorname{Im}\{x\}$ denotes the imaginary part of $x$. The inverse tangent operation in (10) is not only prohibitively complex but also one of the major energy-consuming processes in the receiver.
Following the result reported in equation (35) of [12], a more complexity efficient estimator can be intuitively specialized as 
Input: $\left\{r_{m, k}\right\}$ : complex baseband chip sample sequence;

$L_{1}$ : sample number for each bit of the actual data;

$L_{2}$ : observation length of the preamble in bit intervals;

$L_{3}$ : number of the autocorrelation sample for $m$-th bit of the preamble;

$K$ : length of the PN sequence;

$J$ : bit number in preamble field;

$L$ : payload length of the PPDU;

$N$ : block length.

Output: $\left(\widehat{\phi}_{m-N+1}, \ldots, \widehat{\phi}_{m-1}, \widehat{\phi}_{m}\right)$ : detection of the actual information phase sequence.

(1) initial $Y=0, K=15$, and $J=32$

(2) for $m=2 ; m \leq L_{2} ; m++$ do

(3) for $k=1 ; k \leq L_{3} ; m++$ do

(4) $\quad Y \longleftarrow Y+r_{m, k} r_{m-1, k}^{*}$

(5) end for

(6) end for

(7) compute the estimation function $g(Y)$ for $\widehat{\varphi}$, where $\widehat{\varphi}=\widehat{\omega T_{c}}$;

(8) for $i=m-N ; i \leq m ; i++$ do

(9) for $k=1 ; k \leq L_{1} ; k++$ do

(10) $\quad \mu_{i, k} \longleftarrow r_{i, k} e^{-j[(i-1) K+k] \widehat{\varphi}}$

(11) end for

(12) end for

(13) for $i=m ; i \geq m-N ; i--$ do

(14) for $j=i-1 ; j \geq m-N ; j--$ do

(15) for $k=1 ; k \leq L_{1} ; k++$ do

(16)

(17)

$A_{m-i, m-j} \longleftarrow A_{m-i, m-j}+\mu_{i, k} \mu_{j, k}^{*}$ end for

(18) end for

(19) end for

(20) compute the metric $\Xi$ for each hypothetical phase sequence;

(21) find a phase sequence $\left(\widehat{\phi}_{m-N+1}, \ldots, \widehat{\phi}_{m-1}, \widehat{\phi}_{m}\right)$ that maximizes the metric $\Xi$;

(22) return $\left(\widehat{\phi}_{m-N+1}, \ldots, \widehat{\phi}_{m-1}, \hat{\phi}_{m}\right)$

Algorithm 2: Framework of the conventional MSDD algorithm.

$$
g\left(Y_{i, i^{\prime}}\right)= \begin{cases}\frac{\operatorname{Im}\left(Y_{i, i^{\prime}}\right)}{\operatorname{Re}\left(Y_{i, i^{\prime}}\right)}, & \text { if } \operatorname{Re}\left(Y_{i, i^{\prime}}\right)>0 \text { and }\left|\operatorname{Re}\left(Y_{i, i^{\prime}}\right)\right| \geq\left|\operatorname{Im}\left(Y_{i, i^{\prime}}\right)\right|, \\ \frac{\pi}{2}-\frac{\operatorname{Re}\left(Y_{i, i^{\prime}}\right)}{\operatorname{Im}\left(Y_{i, i^{\prime}}\right)}, & \text { if } \operatorname{Im}\left(Y_{i, i^{\prime}}\right)>0 \text { and }\left|\operatorname{Re}\left(Y_{i, i^{\prime}}\right)\right|<\left|\operatorname{Im}\left(Y_{i, i^{\prime}}\right)\right|, \\ -\pi+\frac{\operatorname{Im}\left(Y_{i, i^{\prime}}\right)}{\operatorname{Re}\left(Y_{i, i^{\prime}}\right)}, & \text { if } \operatorname{Re}\left(Y_{i, i^{\prime}}\right)<0 \text { and }\left|\operatorname{Re}\left(Y_{i, i^{\prime}}\right)\right| \geq\left|\operatorname{Im}\left(Y_{i, i^{\prime}}\right)\right|, \\ -\frac{\pi}{2}-\frac{\operatorname{Re}\left(Y_{i, i^{\prime}}\right)}{\operatorname{Im}\left(Y_{i, i^{\prime}}\right)}, & \text { if } \operatorname{Im}\left(Y_{i, i^{\prime}}\right)<0 \text { and }\left|\operatorname{Re}\left(Y_{i, i^{\prime}}\right)\right|<\left|\operatorname{Im}\left(Y_{i, i^{\prime}}\right)\right| .\end{cases}
$$




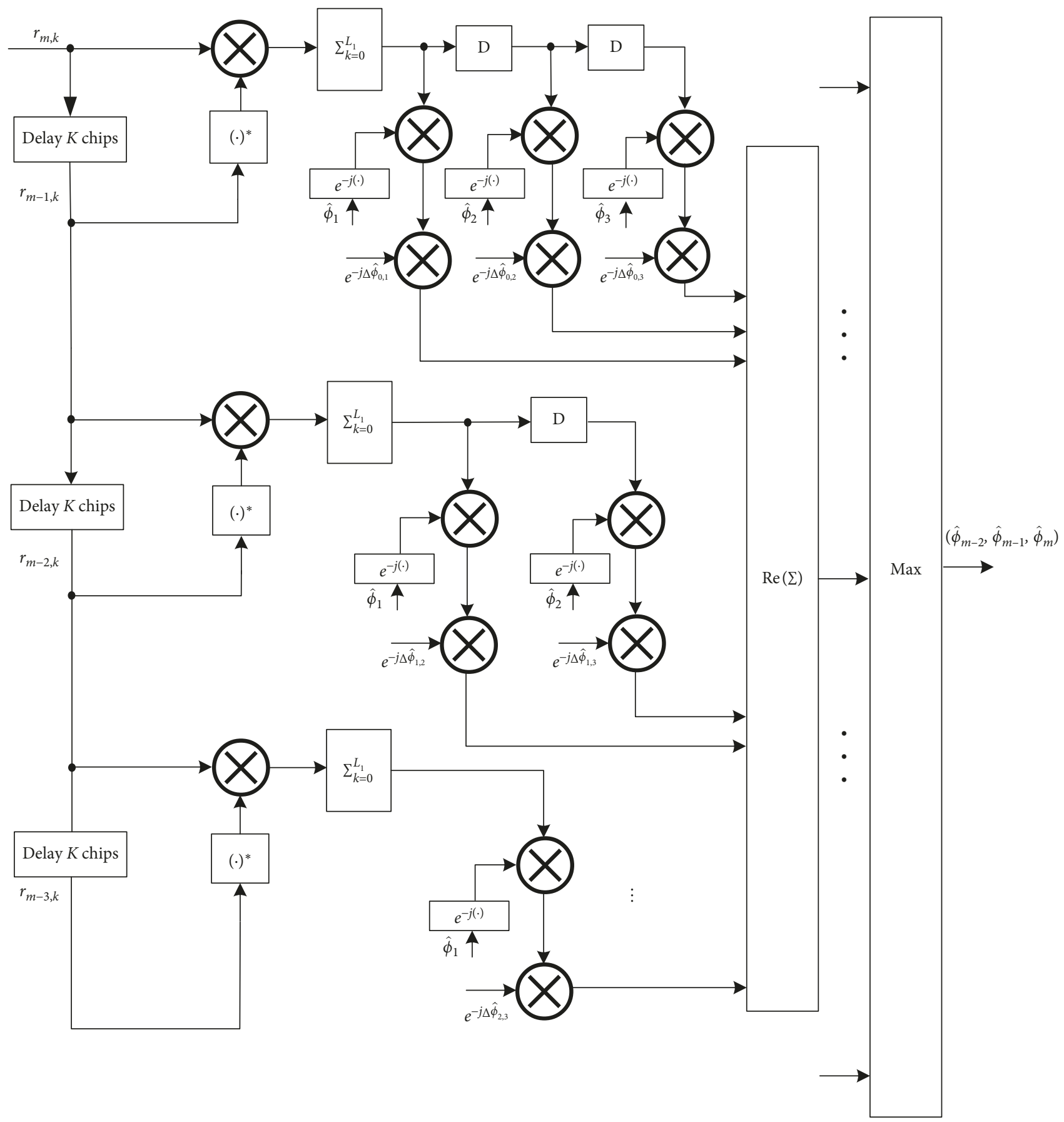

FIgURE 2: The implementation structure of new MSDD for $N=4$. 


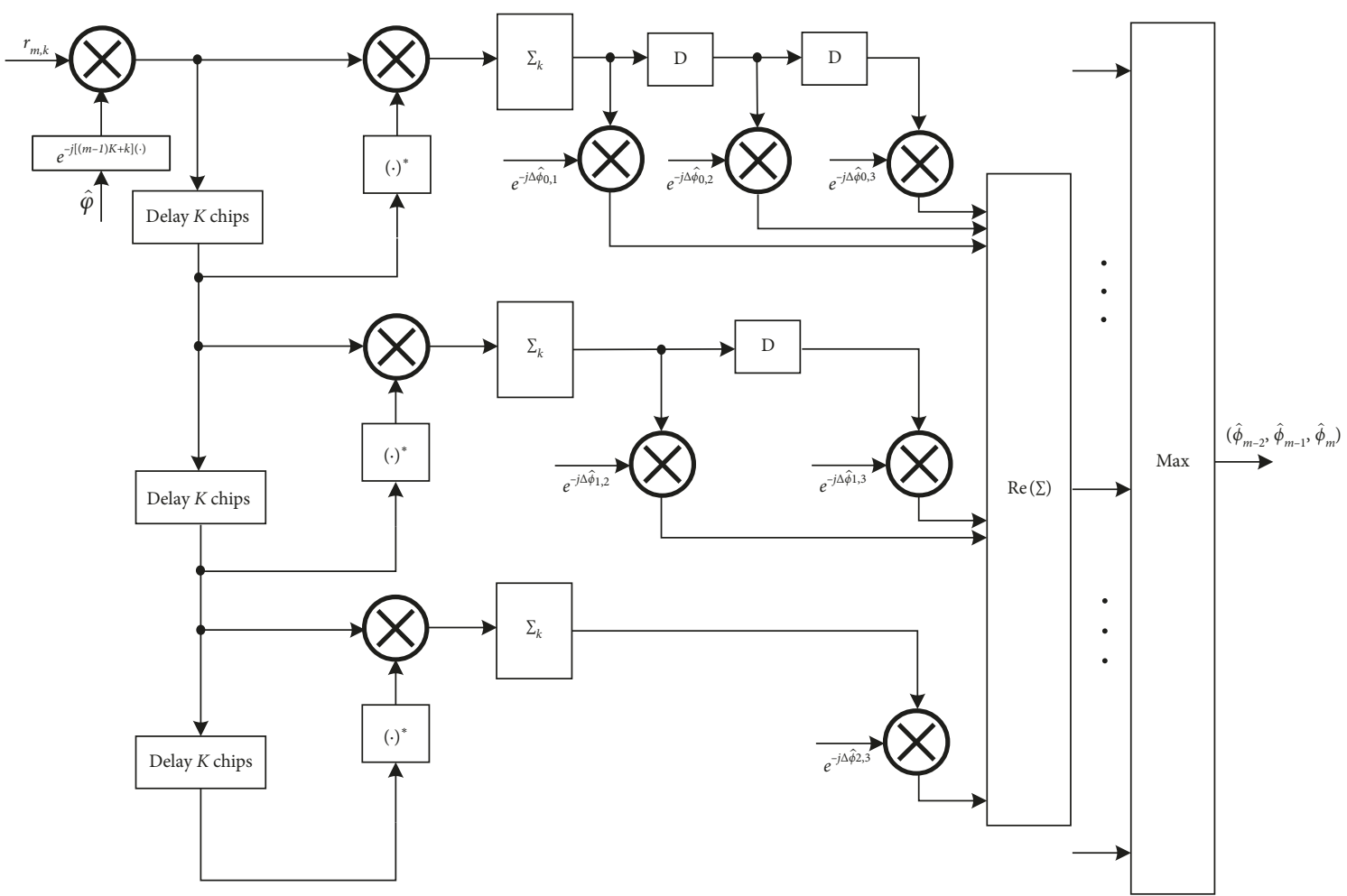

FIgURE 3: The implementation structure of conventional MSDD for $N=4$.

Clearly, the method given in equation (36) of [12] can also be used for estimation. In particular, (4) is now given by

$$
g\left(Y_{i, i^{\prime}}\right)=\left\{\begin{array}{cc}
0, & \text { if } \operatorname{Re}\left(Y_{i, i^{\prime}}\right)>0 \text { and }\left|\operatorname{Re}\left(Y_{i, i^{\prime}}\right)\right| \geq\left|\operatorname{Im}\left(Y_{i, i^{\prime}}\right)\right|, \\
\frac{\pi}{2}, & \text { if } \operatorname{Im}\left(Y_{i, i^{\prime}}\right)>0 \text { and }\left|\operatorname{Re}\left(Y_{i, i^{\prime}}\right)\right|<\left|\operatorname{Im}\left(Y_{i, i^{\prime}}\right)\right|, \\
-\pi, & \text { if } \operatorname{Re}\left(Y_{i, i^{\prime}}\right)<0 \text { and }\left|\operatorname{Re}\left(Y_{i, i^{\prime}}\right)\right| \geq\left|\operatorname{Im}\left(Y_{i, i^{\prime}}\right)\right|, \\
-\frac{\pi}{2}, & \text { if } \operatorname{Im}\left(Y_{i, i^{\prime}}\right)<0 \text { and }\left|\operatorname{Re}\left(Y_{i, i^{\prime}}\right)\right|<\left|\operatorname{Im}\left(Y_{i, i^{\prime}}\right)\right| .
\end{array}\right.
$$

Furthermore, following the result in [12], (4) can be given by

$$
g\left(Y_{i, i^{\prime}}\right)= \begin{cases}\frac{\operatorname{Im}\left(Y_{i, i^{\prime}}\right)}{\left|Y_{i, i^{\prime}}\right|}, & \text { if } \operatorname{Re}\left(Y_{i, i^{\prime}}\right) \geq 0, \\ \pi-\frac{\operatorname{Im}\left(Y_{i, i^{\prime}}\right)}{\left|Y_{i, i^{\prime}}\right|}, & \text { if } \operatorname{Re}\left(Y_{i, i^{\prime}}\right)<0,\end{cases}
$$

over slow Rayleigh and Rician fading channels and further simplified as

$$
g\left(Y_{i, i^{\prime}}\right)= \begin{cases}\operatorname{Im}\left(Y_{i, i^{\prime}}\right), & \text { if } \operatorname{Re}\left(Y_{i, i^{\prime}}\right) \geq 0, \\ \pi-\operatorname{Im}\left(Y_{i, i^{\prime}}\right), & \text { if } \operatorname{Re}\left(Y_{i, i^{\prime}}\right)<0\end{cases}
$$

in pure AWGN channel. Here, $\left|Y_{i, i^{\prime}}\right|$ denotes the magnitude of $Y_{i, i^{\prime}}$. 
We remind the reader that all the estimators given in this section except (14) are feasible under fading channels. However, no CSI about the fading is needed.

\section{Estimation Scheme over Slow Fading Channels}

In this section, a new estimation approach for slow fading channel is considered. We give the detailed derivation in the following. First, we borrow the idea in our previous work [15] and express the phase of the measurement $Y_{i, i^{\prime}}$ as

$$
\angle Y_{i, i^{\prime}}= \begin{cases}\sin ^{-1}\left(\frac{\operatorname{Im}\left(Y_{i, i^{\prime}}\right)}{\sqrt{\operatorname{Re}\left(Y_{i, i^{\prime}}\right)^{2}+\operatorname{Im}\left(Y_{i, i^{\prime}}\right)^{2}}}\right), & \text { if } \operatorname{Re}\left(Y_{i, i^{\prime}}\right)>0 \text { and }\left|\operatorname{Re}\left(Y_{i, i^{\prime}}\right)\right| \geq\left|\operatorname{Im}\left(Y_{i, i^{\prime}}\right)\right|, \\ \frac{\pi}{2}-\sin ^{-1}\left(\frac{\operatorname{Re}\left(Y_{i, i^{\prime}}\right)}{\sqrt{\operatorname{Re}\left(Y_{i, i^{\prime}}\right)^{2}+\operatorname{Im}\left(Y_{i, i^{\prime}}\right)^{2}}}\right), & \text { if } \operatorname{Im}\left(Y_{i, i^{\prime}}\right)>0 \text { and }\left|\operatorname{Re}\left(Y_{i, i^{\prime}}\right)\right|<\left|\operatorname{Im}\left(Y_{i, i^{\prime}}\right)\right|, \\ -\pi-\sin ^{-1}\left(\frac{\operatorname{Im}\left(Y_{i, i^{\prime}}\right)}{\sqrt{\operatorname{Re}\left(Y_{i, i^{\prime}}\right)^{2}+\operatorname{Im}\left(Y_{i, i^{\prime}}\right)^{2}}}\right), & \text { if } \operatorname{Re}\left(Y_{i, i^{\prime}}\right)<0 \text { and }\left|\operatorname{Re}\left(Y_{i, i^{\prime}}\right)\right| \geq\left|\operatorname{Im}\left(Y_{i, i^{\prime}}\right)\right|, \\ -\frac{\operatorname{Re}\left(Y_{i, i^{\prime}}\right)}{2}+\sin ^{-1}\left(\frac{1}{\sqrt{\operatorname{Re}\left(Y_{i, i^{\prime}}\right)^{2}+\operatorname{Im}\left(Y_{i, i^{\prime}}\right)^{2}}}\right), & \text { if } \operatorname{Im}\left(Y_{i, i^{\prime}}\right)<0 \text { and }\left|\operatorname{Re}\left(Y_{i, i^{\prime}}\right)\right|<\left|\operatorname{Im}\left(Y_{i, i^{\prime}}\right)\right| .\end{cases}
$$

Considering that both $\left|\operatorname{Im}\left(Y_{i, i^{\prime}}\right)\right|$ and $\left|\operatorname{Re}\left(Y_{i, i^{\prime}}\right)\right|$ are no more than $\sqrt{\operatorname{Re}\left(Y_{i, i^{\prime}}\right)^{2}+\operatorname{Im}\left(Y_{i, i^{\prime}}\right)^{2}}$, we can immediately simplify (15), and develop a CFOE estimator for the proposed MSDD scheme as follows:

$$
\begin{aligned}
& \text { if } \operatorname{Re}\left(Y_{i, i^{\prime}}\right)>0 \text { and }\left|\operatorname{Re}\left(Y_{i, i^{\prime}}\right)\right| \geq\left|\operatorname{Im}\left(Y_{i, i^{\prime}}\right)\right| \text {, } \\
& \text { if } \operatorname{Im}\left(Y_{i, i^{\prime}}\right)>0 \text { and }\left|\operatorname{Re}\left(Y_{i, i^{\prime}}\right)\right|<\left|\operatorname{Im}\left(Y_{i, i^{\prime}}\right)\right| \text {, } \\
& \text { if } \operatorname{Re}\left(Y_{i, i^{\prime}}\right)<0 \text { and }\left|\operatorname{Re}\left(Y_{i, i^{\prime}}\right)\right| \geq\left|\operatorname{Im}\left(Y_{i, i^{\prime}}\right)\right| \text {, } \\
& \text { if } \operatorname{Im}\left(Y_{i, i^{\prime}}\right)<0 \text { and }\left|\operatorname{Re}\left(Y_{i, i^{\prime}}\right)\right|<\left|\operatorname{Im}\left(Y_{i, i^{\prime}}\right)\right| \text {. }
\end{aligned}
$$


Two multiplication operations and a division operation are still required to achieve the time-varying offset term $\operatorname{Im}\left(Y_{i, i^{\prime}}\right) / \sqrt{\operatorname{Re}\left(Y_{i, i^{\prime}}\right)^{2}+\operatorname{Im}\left(Y_{i, i^{\prime}}\right)^{2}} \quad$ or $\operatorname{Re}\left(Y_{i, i^{\prime}}\right) /$

$$
\begin{aligned}
\sqrt{\operatorname{Re}\left(Y_{i, i^{\prime}}\right)^{2}+\operatorname{Im}\left(Y_{i, i^{\prime}}\right)^{2}} & =\left|Y_{i, i^{\prime}}\right|=\left.|| h\right|^{2} e^{j K \omega T_{c}}+\eta\left(i^{\prime}-i\right) \mid \\
& \approx|h|^{2} .
\end{aligned}
$$

$\sqrt{\operatorname{Re}\left(Y_{i, i^{\prime}}\right)^{2}+\operatorname{Im}\left(Y_{i, i^{\prime}}\right)^{2}}$ as indicated in (16), and should be avoided as much as possible in WSNs [32].

Under the slow fading channel with perfect CSI and if the integrated noise term is sufficient small, we have

$$
g\left(Y_{i, i^{\prime}}\right)= \begin{cases}\frac{\operatorname{Im}\left(Y_{i, i^{\prime}}\right)}{|h|^{2}}, & \text { if } \operatorname{Re}\left(Y_{i, i^{\prime}}\right)>0 \text { and }\left|\operatorname{Re}\left(Y_{i, i^{\prime}}\right)\right| \geq\left|\operatorname{Im}\left(Y_{i, i^{\prime}}\right)\right|, \\ \frac{\pi}{2}-\frac{\operatorname{Re}\left(Y_{i, i^{\prime}}\right)}{|h|^{2}}, & \text { if } \operatorname{Im}\left(Y_{i, i^{\prime}}\right)>0 \text { and }\left|\operatorname{Re}\left(Y_{i, i^{\prime}}\right)\right|<\left|\operatorname{Im}\left(Y_{i, i^{\prime}}\right)\right|, \\ -\pi-\frac{\operatorname{Im}\left(Y_{i, i^{\prime}}\right)}{|h|^{2}}, & \text { if } \operatorname{Re}\left(Y_{i, i^{\prime}}\right)<0 \text { and }\left|\operatorname{Re}\left(Y_{i, i^{\prime}}\right)\right| \geq\left|\operatorname{Im}\left(Y_{i, i^{\prime}}\right)\right|, \\ -\frac{\pi}{2}+\frac{\operatorname{Re}\left(Y_{i, i^{\prime}}\right)}{|h|^{2}}, & \text { if } \operatorname{Im}\left(Y_{i, i^{\prime}}\right)<0 \text { and }\left|\operatorname{Re}\left(Y_{i, i^{\prime}}\right)\right|<\left|\operatorname{Im}\left(Y_{i, i^{\prime}}\right)\right| .\end{cases}
$$

Until now, the number of multiplication operation has been reduced. Furthermore, for pure AWGN channel, we have

$$
\sqrt{\operatorname{Re}\left(Y_{i, i^{\prime}}\right)^{2}+\operatorname{Im}\left(Y_{i, i^{\prime}}\right)^{2}} \approx|h|^{2}=1
$$

$$
g\left(Y_{i, i^{\prime}}\right)=\left\{\begin{array}{cc}
\operatorname{Im}\left(Y_{i, i^{\prime}}\right), \quad \text { if } \operatorname{Re}\left(Y_{i, i^{\prime}}\right)>0 \text { and }\left|\operatorname{Re}\left(Y_{i, i^{\prime}}\right)\right| \geq\left|\operatorname{Im}\left(Y_{i, i^{\prime}}\right)\right|, \\
\frac{\pi}{2}-\operatorname{Re}\left(Y_{i, i^{\prime}}\right), \quad \text { if } \operatorname{Im}\left(Y_{i, i^{\prime}}\right)>0 \text { and }\left|\operatorname{Re}\left(Y_{i, i^{\prime}}\right)\right|<\left|\operatorname{Im}\left(Y_{i, i^{\prime}}\right)\right|, \\
-\pi-\operatorname{Im}\left(Y_{i, i^{\prime}}\right), & \text { if } \operatorname{Re}\left(Y_{i, i^{\prime}}\right)<0 \text { and }\left|\operatorname{Re}\left(Y_{i, i^{\prime}}\right)\right| \geq\left|\operatorname{Im}\left(Y_{i, i^{\prime}}\right)\right|, \\
-\frac{\pi}{2}+\operatorname{Re}\left(Y_{i, i^{\prime}}\right), & \text { if } \operatorname{Im}\left(Y_{i, i^{\prime}}\right)<0 \text { and }\left|\operatorname{Re}\left(Y_{i, i^{\prime}}\right)\right|<\left|\operatorname{Im}\left(Y_{i, i^{\prime}}\right)\right| .
\end{array}\right.
$$

In this context, a multiplication operation and a division operation are further removed, and we arrive at the estimation scheme given in [15]. In Table 1, we describe all of the estimators for our proposed MSDD scheme in detail. as shown in Figure 3 of [12]. In this context, (18) can be further simplified as 
TABLE 1: Comparison of different estimation schemes.

\begin{tabular}{lccc}
\hline Estimator & Subspace number & Involved mathematical approximation & Channel condition \\
\hline$(10)$ & 2 & No & Fading, no CSI \\
$(11)$ & 4 & $\tan ^{-1}(x) \approx x$ & Fading, no CSI \\
$(12)$ & 4 & $\tan ^{-1}(x) \approx 0$ & Fading, no CSI \\
$(13)$ & 2 & $\sin ^{-1}(x) \approx x$ & Fading, no CSI \\
$(14)$ & 2 & $\sin ^{-1}(x) \approx x, \sqrt{\operatorname{Re}\left(Y_{i, i^{\prime}}\right)^{2}+\operatorname{Im}\left(Y_{i, i^{\prime}}\right)^{2}} \approx 1$ & Pure AWGN \\
$(16)$ & 4 & $\sin ^{-1}(x) \approx x$ & Fading, no CSI \\
$(18)$ & 4 & $\sin ^{-1}(x) \approx x, \sqrt{\operatorname{Re}\left(Y_{i, i^{\prime}}\right)^{2}+\operatorname{Im}\left(Y_{i, i^{\prime}}\right)^{2}} \approx|h|^{2}$ & Fading, perfect CSI \\
$(20)$ & & $\sin ^{-1}(x) \approx x, \sqrt{\operatorname{Re}\left(Y_{i, i^{\prime}}\right)^{2}+\operatorname{Im}\left(Y_{i, i^{\prime}}\right)^{2}} \approx 1$ & Pure AWGN \\
\hline
\end{tabular}

TABle 2: Parameters used in the simulations.

\begin{tabular}{lc}
\hline Parameters & Detailed description \\
\hline Channel condition & Slow fading or pure AWGN \\
Power of the complex AWGN & NorsN \\
Power of Rayleigh fading channel & Nit-level MSDD \\
Detection scheme & Precompensation or postcompensation \\
Compensation scheme & Perfect \\
Timing synchronisation & $1+x+x^{4}$ \\
Generator polynomial of the PN code & 160 \\
Payload length of the PPDU (bits) & 924 \\
Carrier frequency $(\mathrm{MHz})$ & $6 \times 10^{5}$ \\
Chip rate (chip/s) & Uniform distribution in $(-\pi, \pi)$ or Wiener process \\
Carrier phase offset $\theta$ (rads) & Symmetric triangular shape in $(-80,80)$ \\
CFO $f$ (ppm) & 15 \\
Sample number $L_{1}$ & 32 \\
Observation length $L_{2}$ & 15 \\
Sample number $L_{3}$ &
\end{tabular}

the corresponding results. All of the parameters used in our simulations are described in detail in Table 2.

\subsection{Detection Performance Influence of the Bit Observation} Window Length over Pure AWGN Channel. As shown in Figures 1 and 2 of [15], the conventional MSDD scheme exhibits serious error floor if equipped with the estimator given in (11). This is also intuitively observed for the estimator given in (12) as depicted in Figure 6 of this work. Surprisingly, unlike the conventional scheme, error floor does not arise in our new MSDD as observed from Figures 4 to 6. Especially, our new MSDD scheme converges at all SNR regions even if we equip it with the most simple estimator given in (12) and increase the observation length $N$ as large as possible (e.g., $N=8$ ) as indicated in Figure 6. In other words, conventional MSDD algorithm is estimator-selective, and the estimator with small error must be implemented to avoid the error floor. However, our improved strategy is estimator-nonselective, and any now available estimator can be configured.

Furthermore, as indicated in Figure 7, if the mathematical approximation in (19) is involved, more and more performance gap will be arisen in conventional MSDD with the increase of $N$. Yet, this is not observed in our new strategy. That is, almost no performance loss is arisen under any observation window length $N$. Therefore, the divisor-free estimator given in (20) is much more suitable for implementation in our new detection scheme. This result can also be confirmed by Figure 4. Finally, the optimum value for the observation length $N$ is 7 to achieve reasonable balance between detection performance and the computational complexity

\subsection{Detection Performance Influence of the Bit Observation} Window Length over Slow Fading Channel. The performance of the MSDD is depicted in Figures 8 and 9, wherein the channel model is set as the slow Rayleigh fading channel with normalized average power. As observed from Figures 8 and 9, the MSDD is also able to achieve a substantial improvement with $N$ as low as three or four. On the other hand, error floor is surprisingly not observed for conventional MSDD with (11) if we increase $N$ to 6 . Error floor is still intuitively observed for the estimators given in (12) and (13). However, no error floor is observed for our new MSDD scheme with any simplified estimator. Thus, our improved strategy is also estimatornonselective over slow fading channel. Finally, as observed from Figure 9, the optimum value for $N$ is 5 for our new MSDD scheme with the estimators in (12) and (13).

6.3. Detection Performance Influence of the Mathematical Approximation Involved in the Estimator. In Figure 10, the 

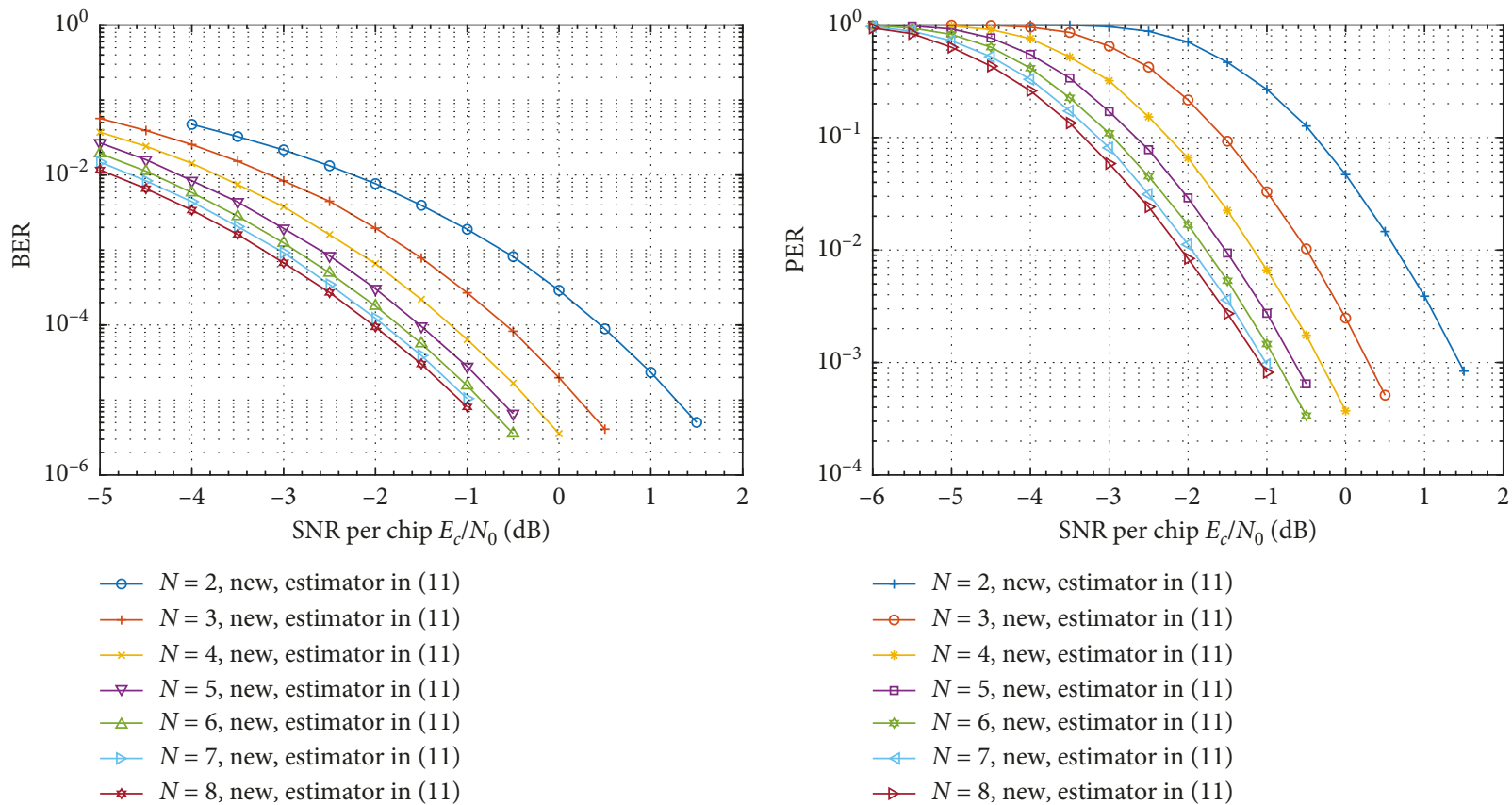

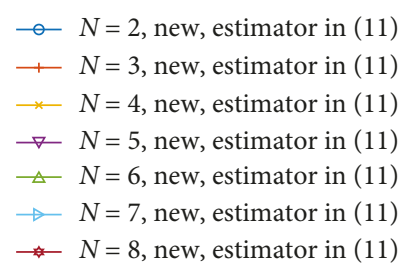

(a) (b)

FIGURE 4: BER and PER performance of the new MSDD scheme with the estimator in (11) over pure AWGN channel. (a) BER for new MSDD; (b) PER for new MSDD.

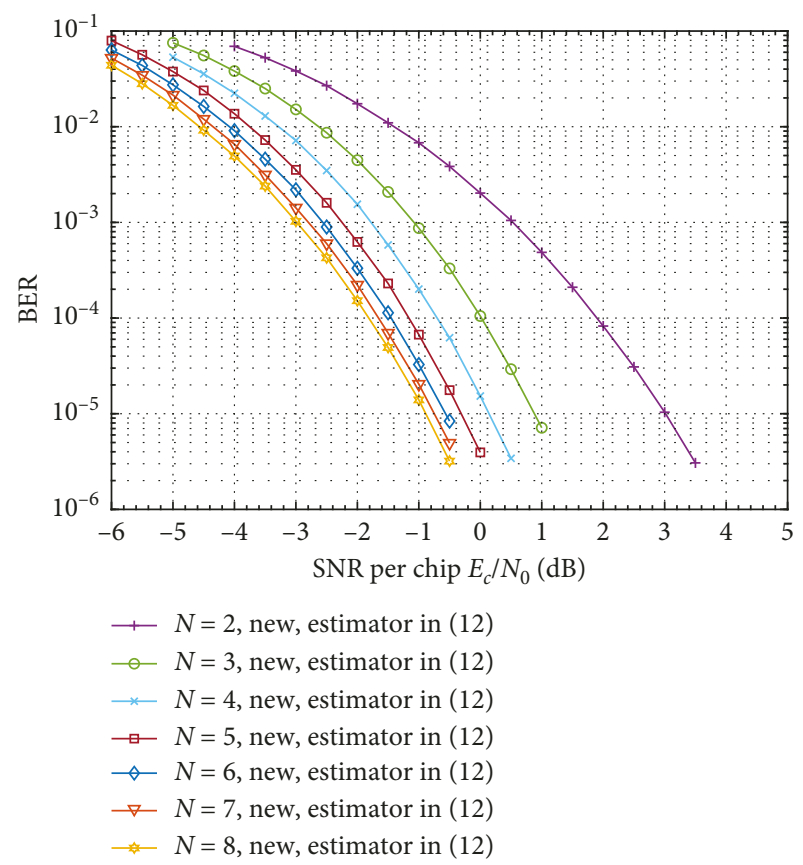

(a)

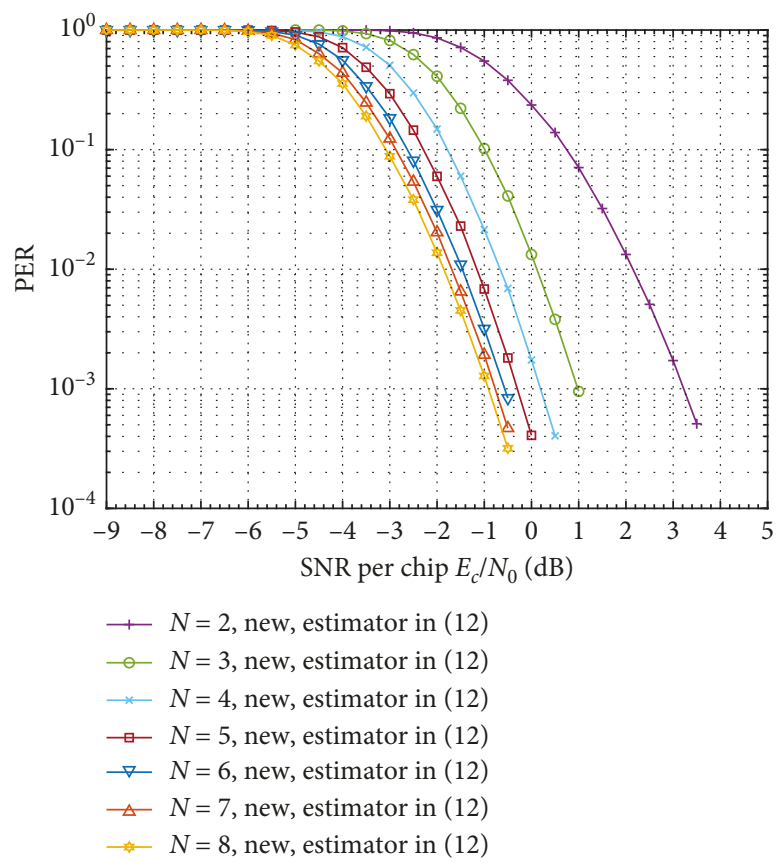

(b)

FIGURE 5: BER and PER performance of the new MSDD scheme with the estimator in (12) over pure AWGN channel. (a) BER for new MSDD; (b) PER for new MSDD.

performance result the involved mathematical approximations have on the new MSDD scheme over pure AWGN channel is given. Referring to Figure 10, for the new detection scheme, numerical results confirm that almost no much performance degradation is observed at all SNR regions especially when we increase $N$, despite that a 

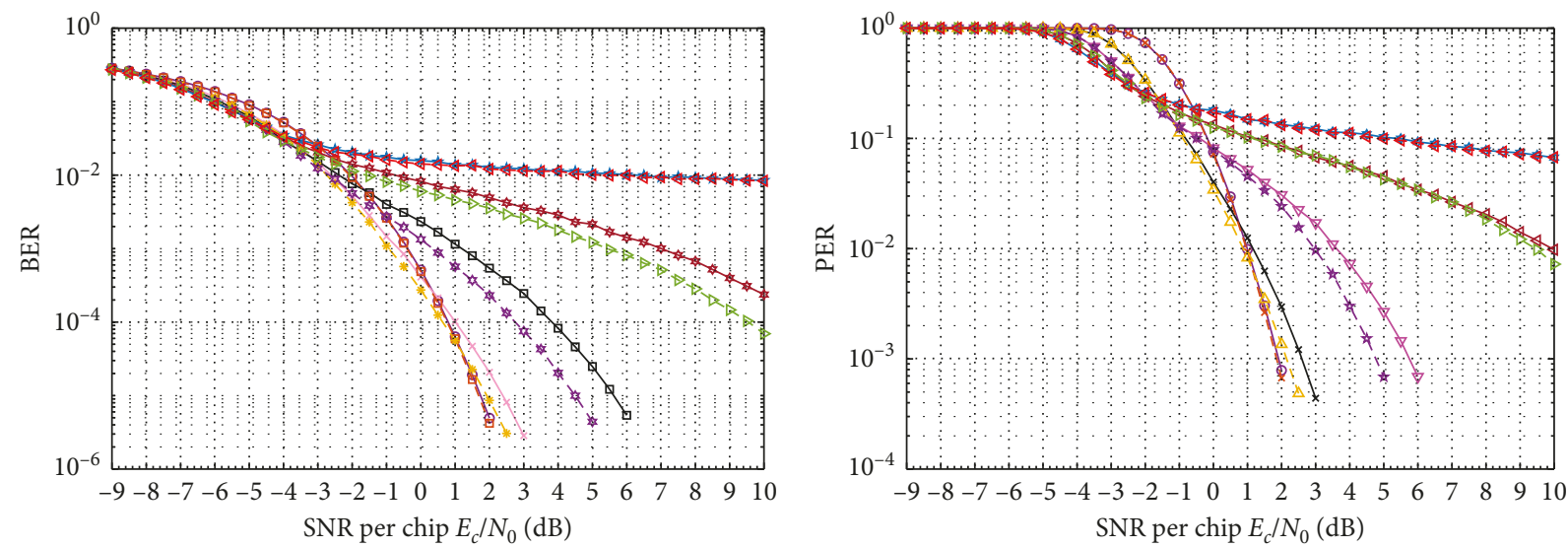

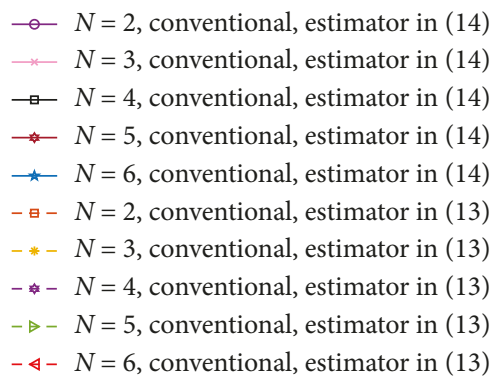

(a)

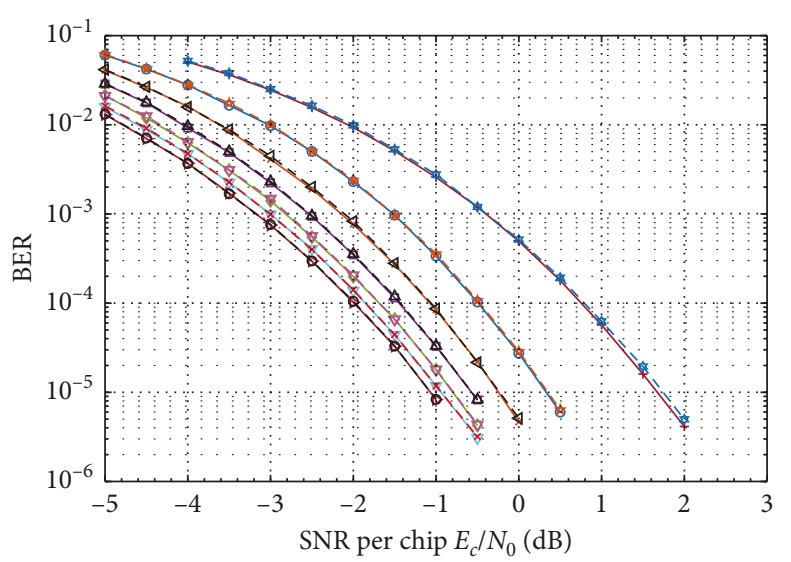

十 $N=2$, new, estimator with (13)

$\rightarrow N=3$, new, estimator with (13)

* $N=4$, new, estimator with (13)

$\rightarrow \quad N=5$, new, estimator with (13)

$\rightarrow N=6$, new, estimator with (13)

$\neg N=7$, new, estimator with (13)

$\rightarrow N=8$, new, estimator with (13)

- $N=2$, new, estimator with (14)

- - $N=3$, new, estimator with (14)

$-\varangle-N=4$, new, estimator with (14)

- $₫-N=5$, new, estimator with (14)

$-\nabla-N=6$, new, estimator with (14)

- * $N=7$, new, estimator with (14)

- $\theta-N=8$, new, estimator with (14)

(c)

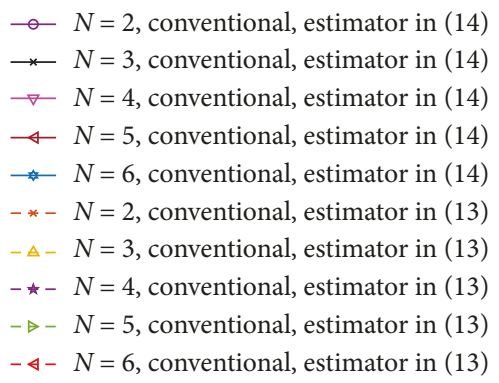

(b)

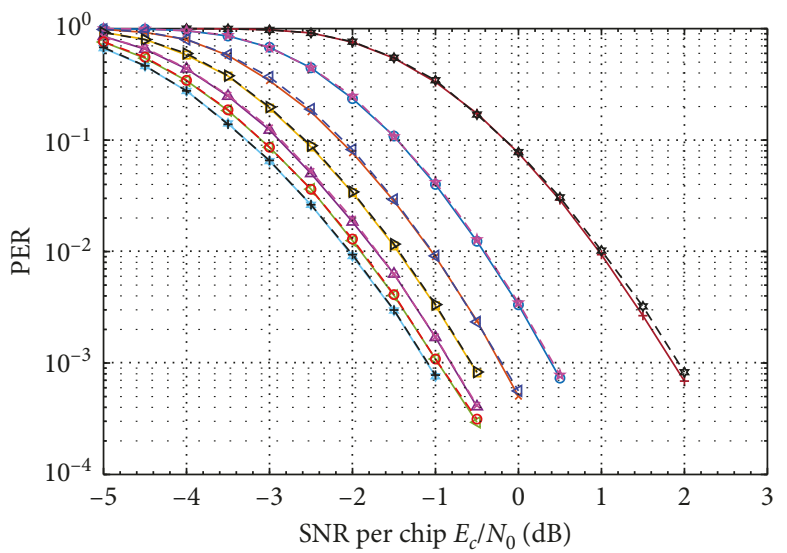

- $N=2$, new, estimator with (13)

- $N=3$, new, estimator with (13)

* $N=4$, new, estimator with (13)

$\rightarrow-N=5$, new, estimator with (13)

$\triangle N=6$, new, estimator with (13)

$\dashv N=7$, new, estimator with (13)

$-N=8$, new, estimator with (13)

- $N=2$, new, estimator with (14)

$-\star-N=3$, new, estimator with (14)

$-\varangle-N=4$, new, estimator with (14)

$\rightarrow-N=5$, new, estimator with (14)

$-*-N=6$, new, estimator with (14)

$-\theta-N=7$, new, estimator with (14)

$-+-N=8$, new, estimator with (14)

(d)

FIGURE 6: Detection performance of the MSDD scheme with estimators given in (13) and (14) over pure AWGN channel. (a) BER for conventional MSDD; (b) PER for conventional MSDD; (c) BER for new MSDD; (d) PER for new MSDD. 

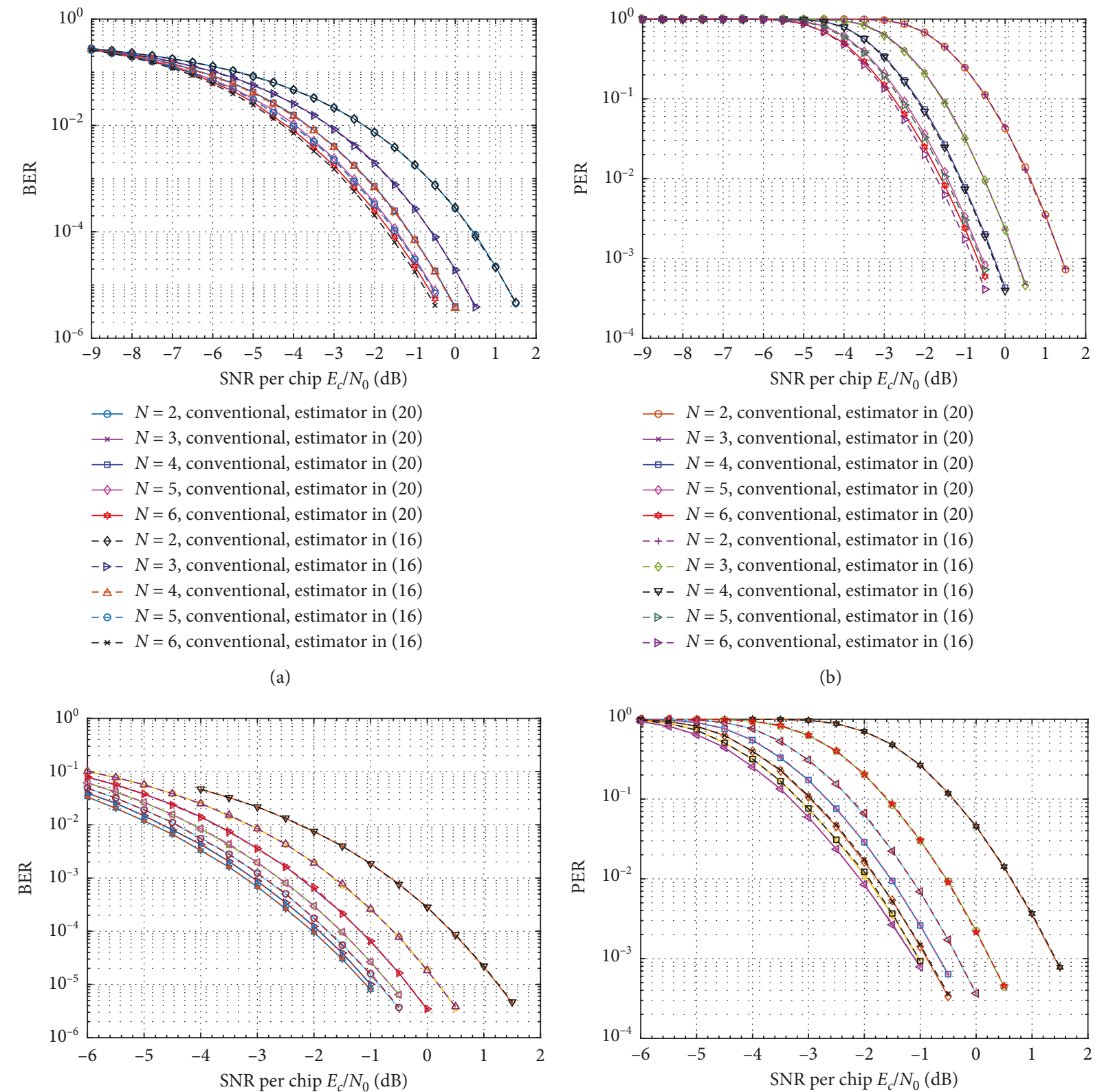

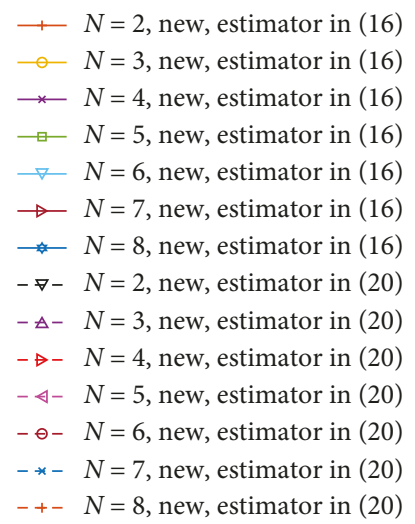

(c)

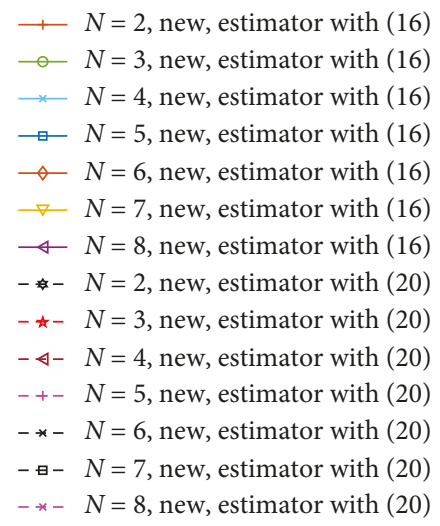

(d)

FIGURE 7: Detection performance of the MSDD scheme with estimators given in (16) and (20) over pure AWGN channel. (a) BER for conventional MSDD; (b) PER for conventional MSDD; (c) BER for new MSDD; (d) PER for new MSDD. 

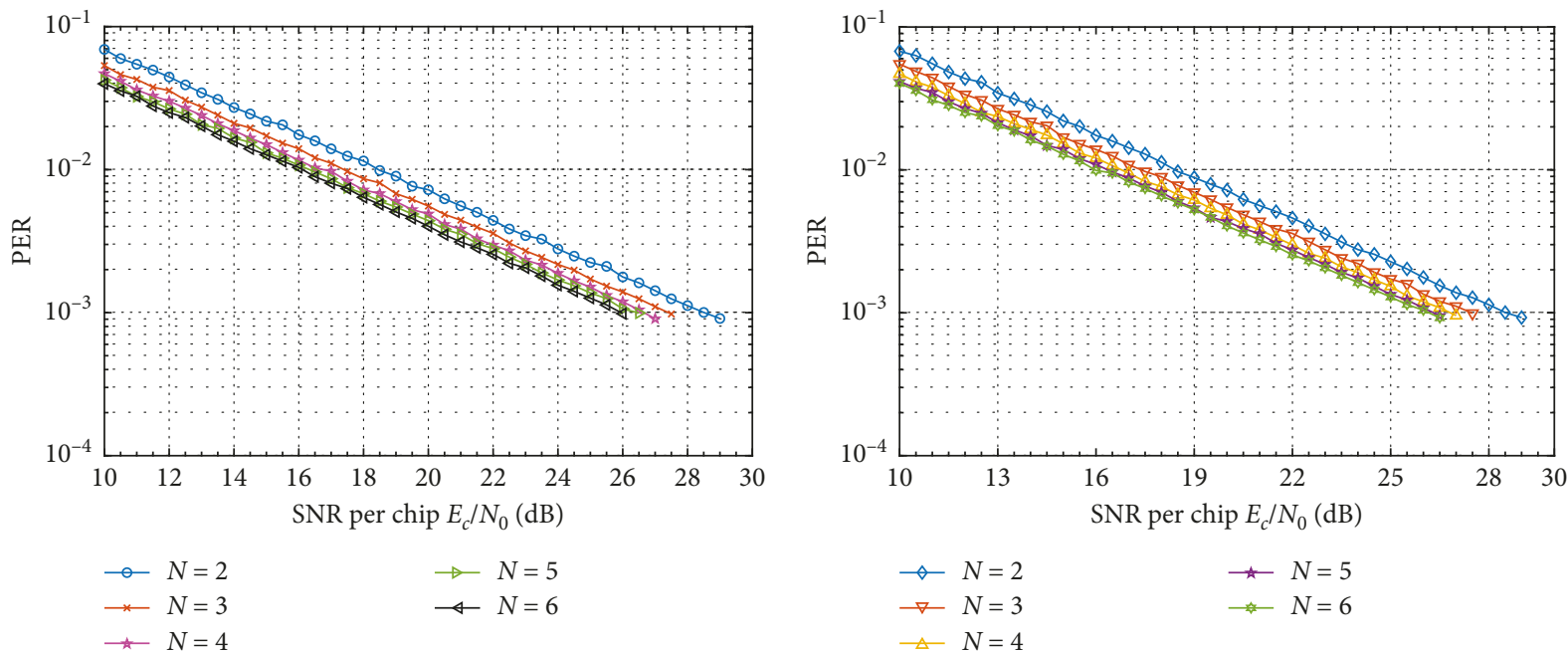

(a)

(b)
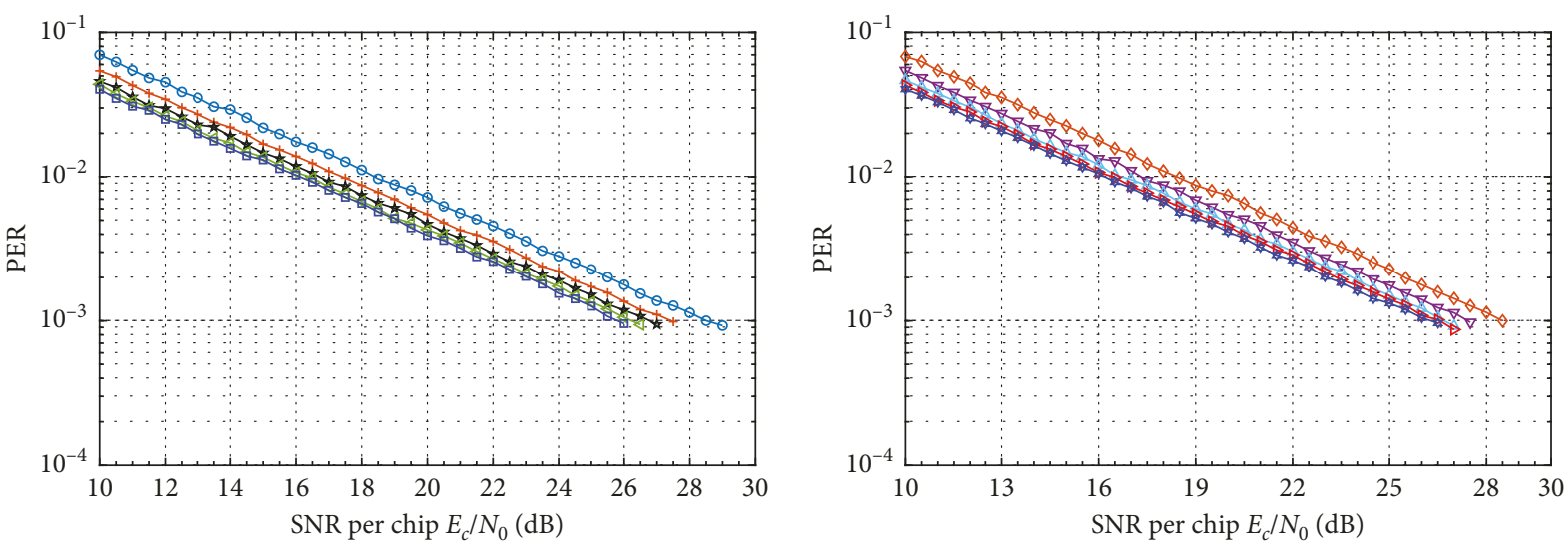

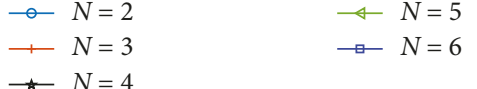

(c)

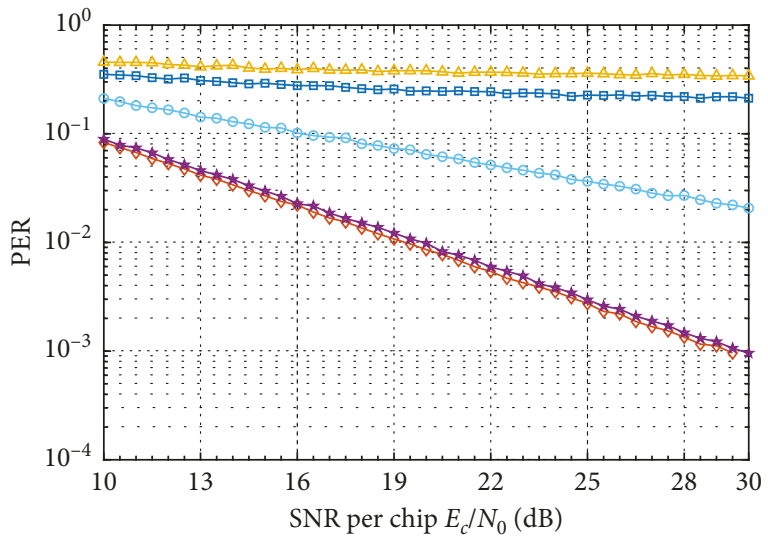

$$
\begin{aligned}
& \multimap N=2 \\
& \star N=3 \\
& \multimap N=4
\end{aligned}
$$

$$
\begin{aligned}
& \rightarrow N=2 \\
& \rightarrow N=3 \\
& \triangle N=4
\end{aligned}
$$

(d)

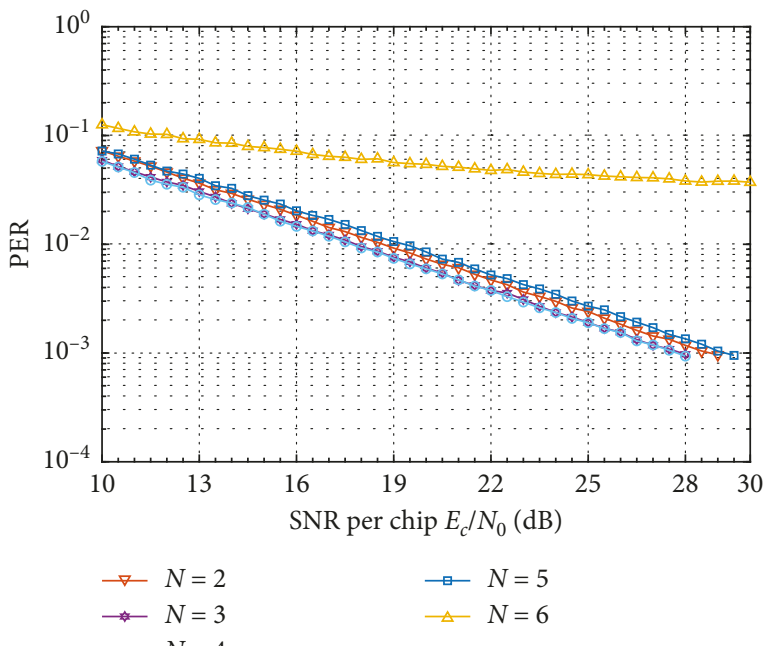

(f)

FIgURE 8: PER performance of conventional MSDD scheme with the different estimators under the normalized slow Rayleigh fading channel. (a) Performance for estimator given in (16); (b) performance for estimator given in (18); (c) performance for estimator given in (10); (d) performance for estimator given in (11); (e) performance for estimator given in (12); (f) performance for estimator given in (13). 


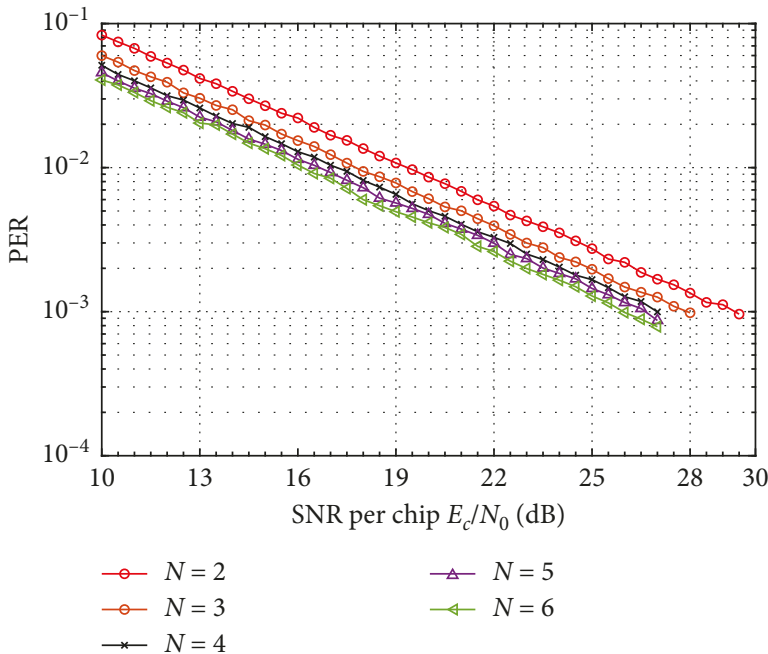

(a)

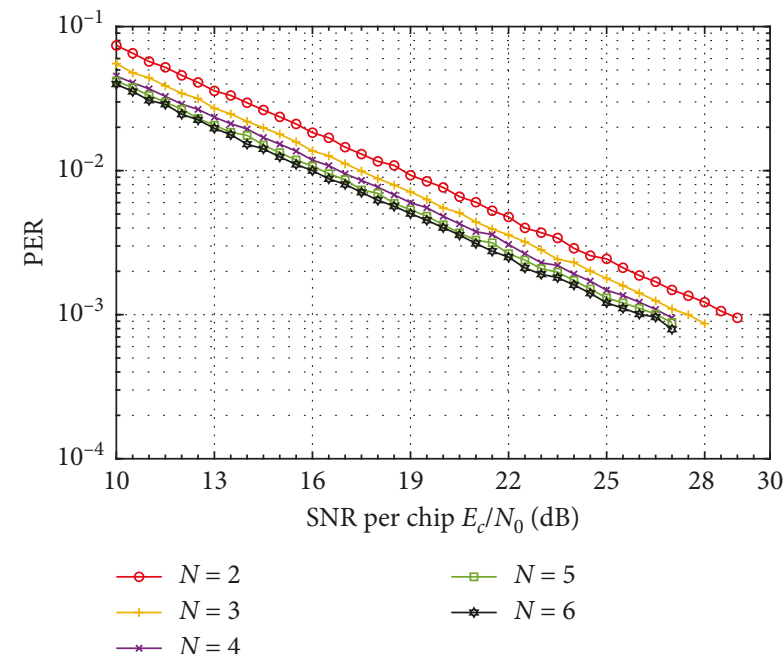

(b)

FIGURE 9: PER performance of the proposed MSDD scheme with the different estimators under the normalized slow Rayleigh fading channel. (a) Performance for estimator given in (12) and (b) performance for estimator given in (13).

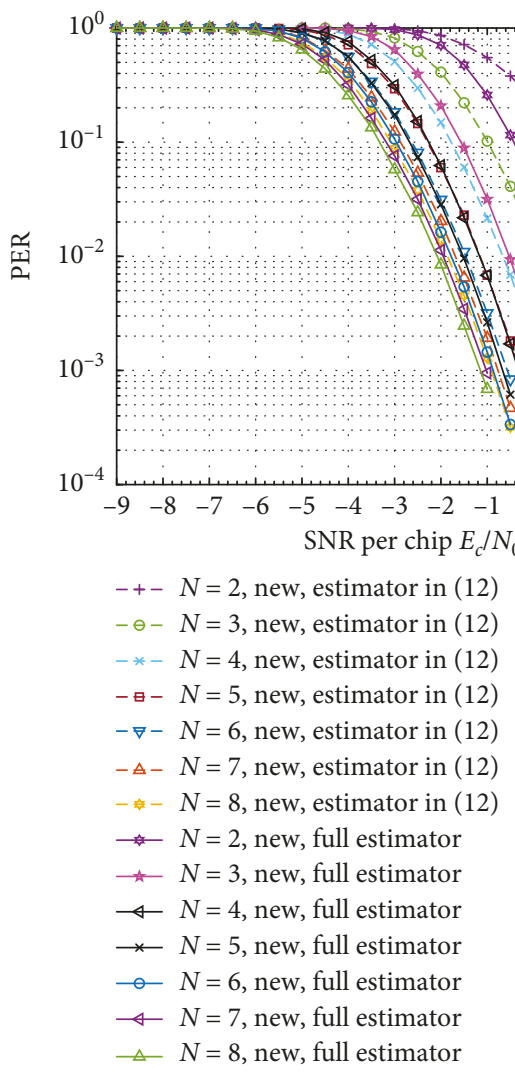

(a)

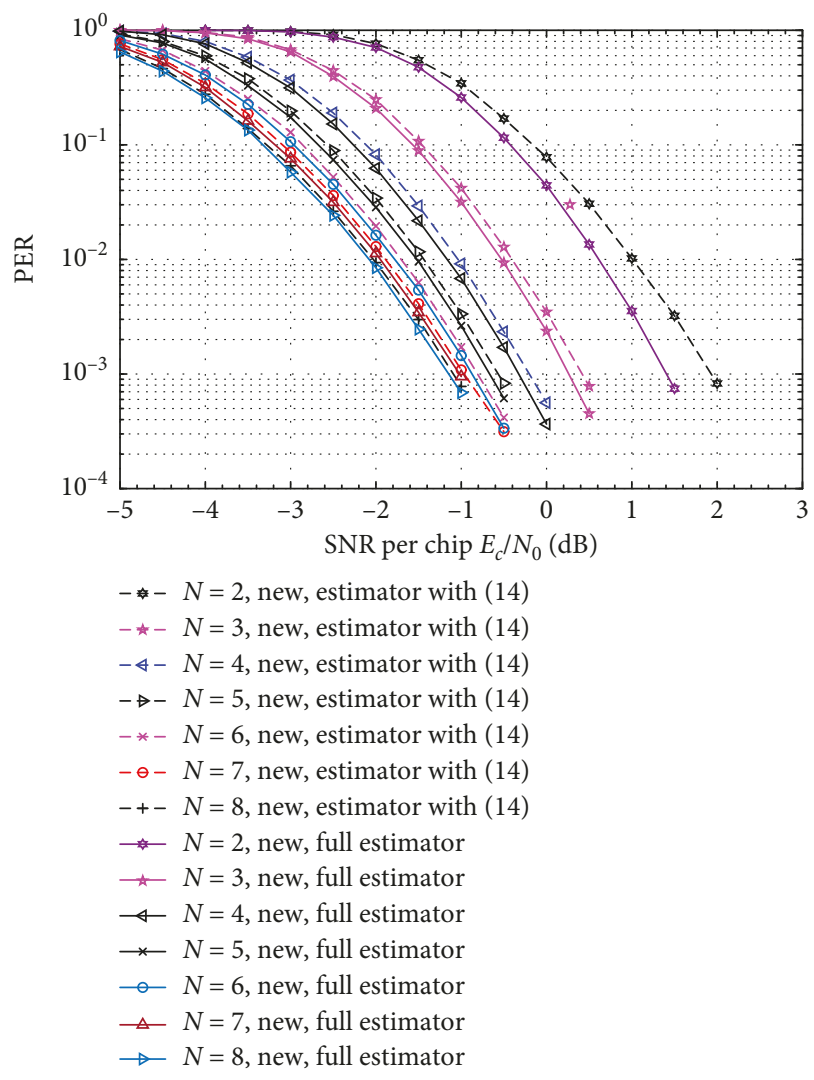

(b)

Figure 10: Continued. 


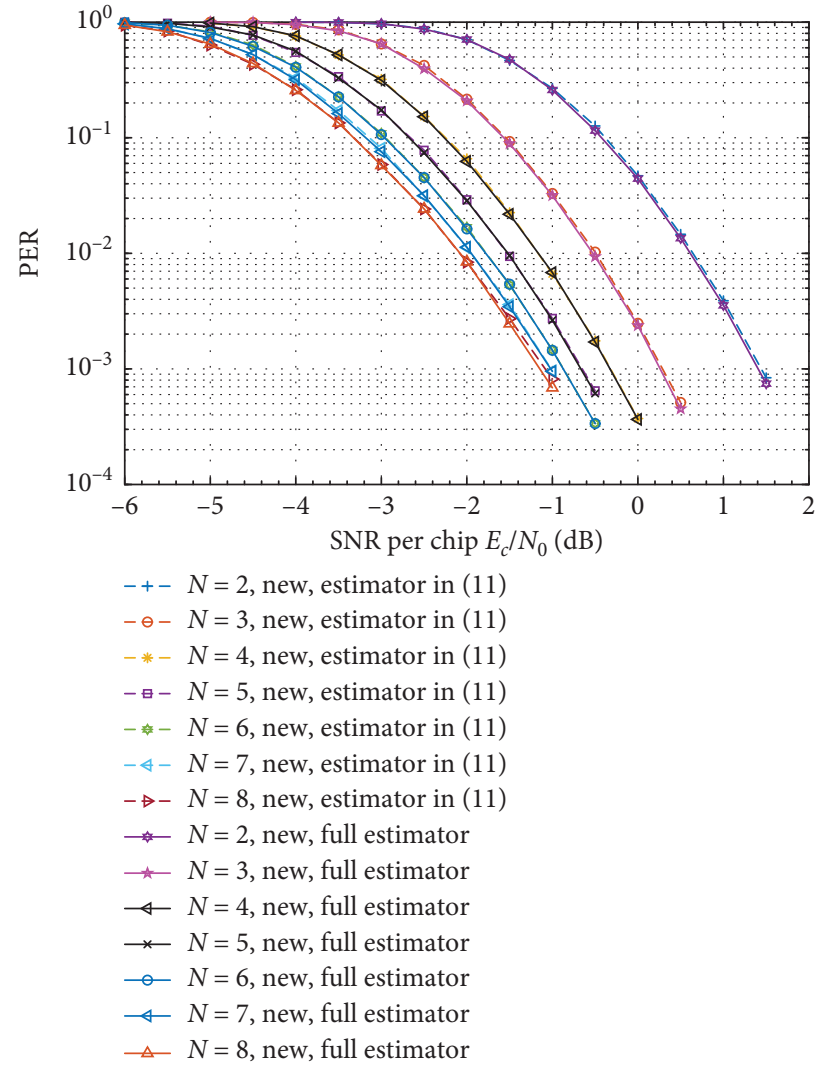

(c)

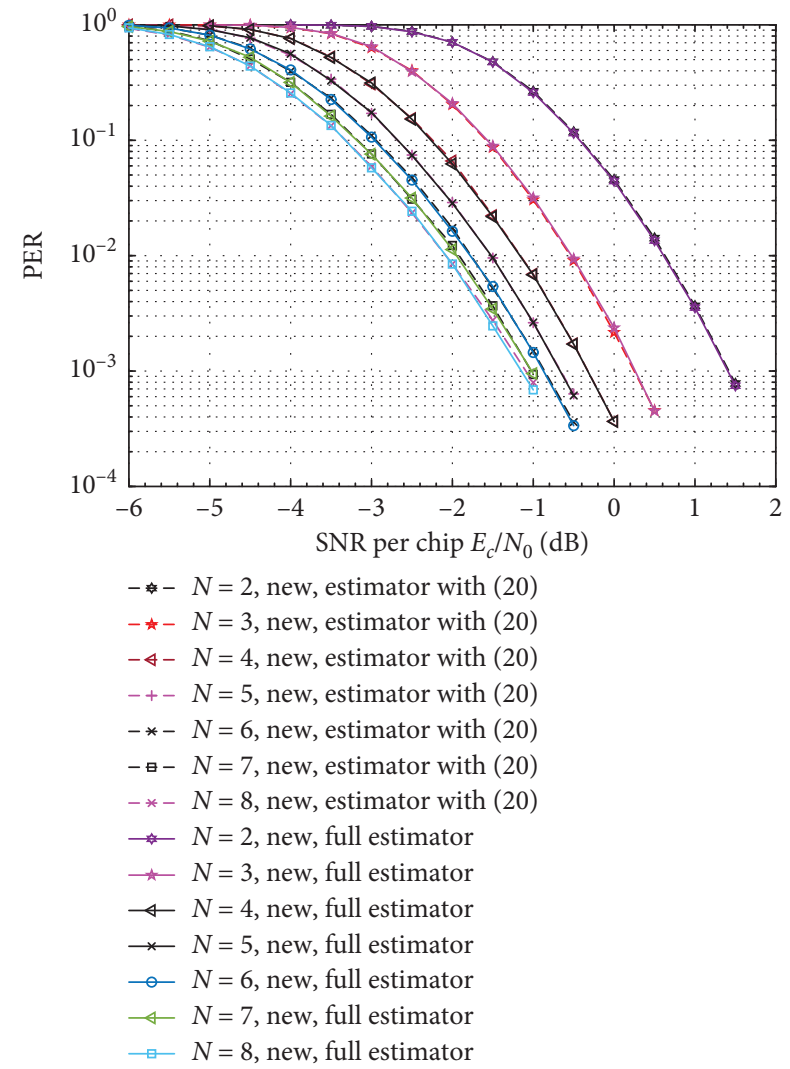

(d)

FIGURe 10: PER performance comparison of the new MSDD scheme with different estimators over pure AWGN channel. (a) Performance for estimators given in (12) and (10); (b) performance for estimators given in (14) and (10); (c) performance for estimators given in (11) and (10); (d) performance for estimators given in (20) and (10).

theoretical basis is still lacked, but the decrease in complexity of the estimator is acquired. Therefore, all of the available complexity efficient estimators are compatible with our proposed MSDD. Similar result can also be observed over slow fading channel, which however is not given here.

\subsection{Detection Performance Comparison for Two Detection} Strategies. In Figures 11 and 12, we give detection evaluation results of the two MSDD strategies over pure AWGN channel. Referring to Figure 11, with full estimator, performance of our new strategy outperforms that of the conventional strategy, although the gap is so small under all observation window lengths. To further exhibit the advantage of our configuration, performances of the new strategy with simplified estimator given in (20) and conventional strategy with full estimator are also given in Figure 11. No much performance degradation is observed. In Figure 12, BER and PER performances of the new strategy with various estimators when $N$ is set to be 8 are given. As shown in Figure 12, our new MSDD can achieve about $2.5 \mathrm{~dB}$ gain from differential and spread spectrum coding when compared with the conventional SDCD scheme for PER of $1 \times 10^{-3}$. The performance gap between the new MSDD and the coherent detection with single differential decoding is no more than $1 \mathrm{~dB}$.
6.5. Transmitter Energy Consumption for New Detection Strategy. We estimate the IEEE BPSK PHY's transmitter in the Atmel AT86RF212B [27]. The consumed transmission energy is given by $E=I V N_{p} / f_{t x}$ [26]. Here, $I$ is the transmission current, the supply voltage $V$ is assumed to be $3 V, N_{p}$ is the chip length of the entire data payload, and the transmission rate is $f_{t x}=6 \times 10^{5}$ chip per second [8].

The consumed transmission current $I$ depends on the distributed transmit energy for each chip symbol [27]. However, invoking the proposed MSDD scheme equipped with the estimator in (18) facilitates reductions of 1.15$2.20 \mathrm{~dB}$ in the distributed transmit energy at PER $=1 \times 10^{-3}$ when the bit observation window length $N$ is set to be 6 , as shown in Table 3. Table 4 provides the amount of transmit energy for each chip symbol consumed by the MSDD scheme, as well as the overall amount of the associated energy savings. As shown in Table 4 and Figure 13, more than $13 \%$ transmit energy for each chip symbol may be saved in transmit-only nodes at PER $=1 \times 10^{-3}$.

Table 5 provides the amount of current consumed in the transmitter of the sensor node when PER $=1 \times 10^{-3}$. As shown in Table 5, reductions of 1.20-2.10 dB in the required transmit energy per chip is also achieved over the pure AWGN channel. Similarly, more than $13 \%$ energy may also be saved. 

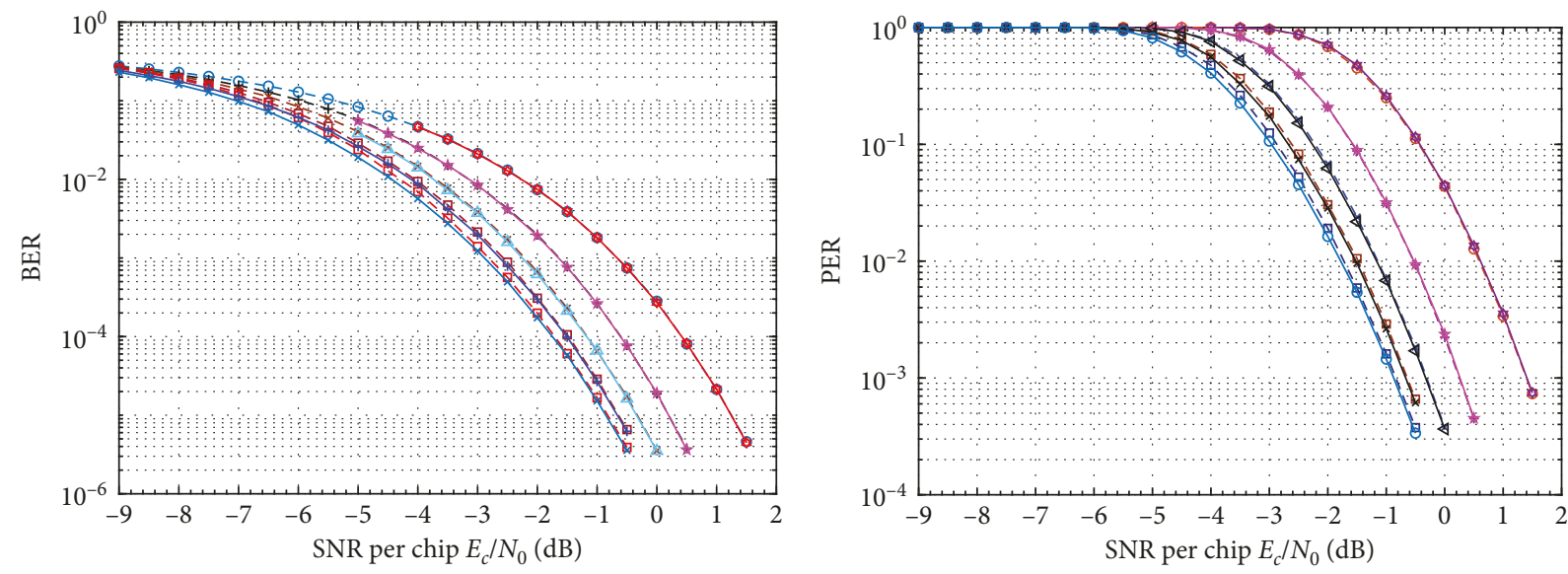

- $\theta-N=2$, conventional, full estimator

-+- $N=3$, conventional, full estimator

-* - $N=4$, conventional, full estimator

- $-N=5$, conventional, full estimator

- $-N=6$, conventional, full estimator

* $N=2$, new, full estimator

* $N=3$, new, full estimator

$\triangle N=4$, new, full estimator

$\longrightarrow N=5$, new, full estimator

$* N=6$, new, full estimator

(a)
- $\theta-N=2$, conventional, full estimator
-+- $N=3$, conventional, full estimator
- * $N=4$, conventional, full estimator
-в- $N=5$, conventional, full estimator
- $-N=6$, conventional, full estimator
* $N=2$, new, full estimator
\# $N=3$, new, full estimator
$\varangle N=4$, new, full estimator
* $N=5$, new, full estimator
$\rightarrow N=6$, new, full estimator

(b)
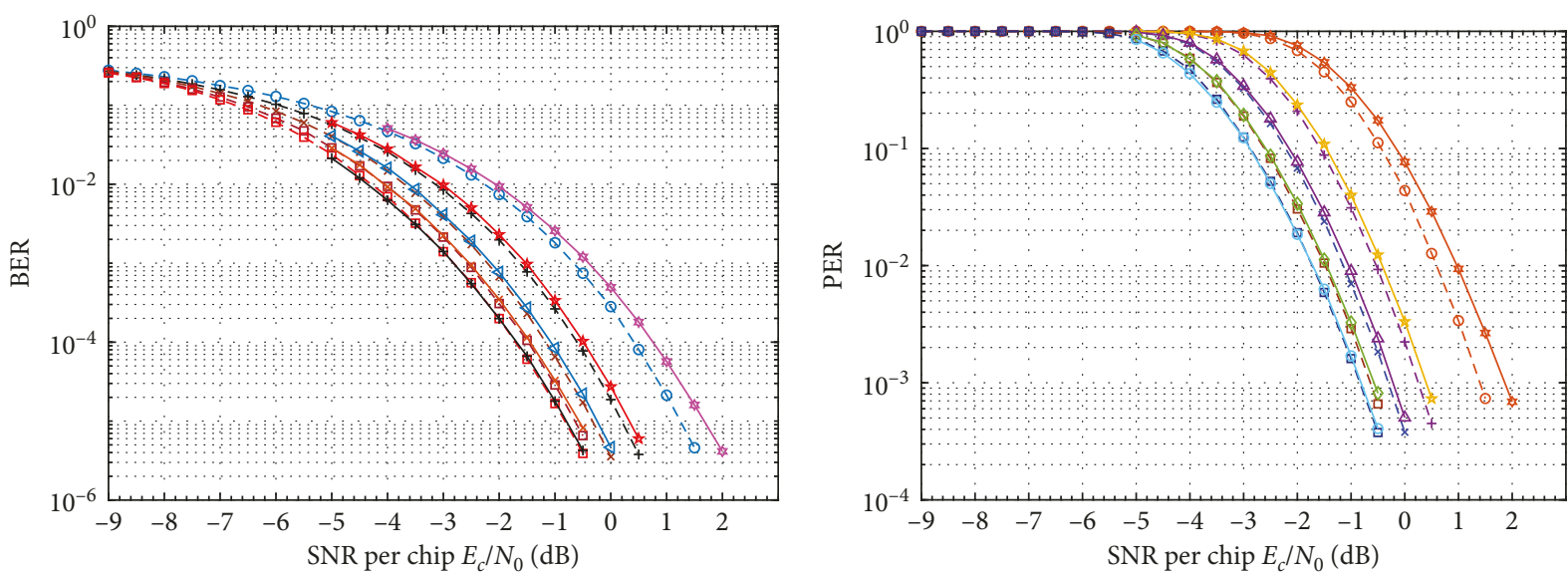

- $\ominus-N=2$, conventional, full estimator

-+ $N=3$, conventional, full estimator

-* - $N=4$, conventional, full estimator

$-\Xi-N=5$, conventional, full estimator

$-\boxminus-N=6$, conventional, full estimator

$\sharp N=2$, new, estimator with (13)

$\star N=3$, new, estimator with (13)

$\triangleleft N=4$, new, estimator with (13)

$\star N=5$, new, estimator with (13)

$\longrightarrow N=6$, new, estimator with (13)

(c)

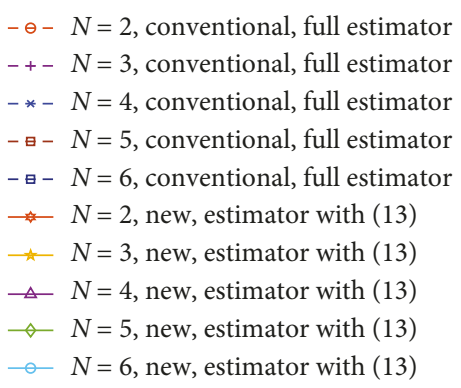

(d)

FIGURE 11: Detection performance comparisons for two detection strategies over pure AWGN channel. (a) BER performance with estimator in (10); (b) PER performance with estimator in (10); (c) BER performance with estimators in (10) and (13); (d) PER performance with estimators in (10) and (13). 


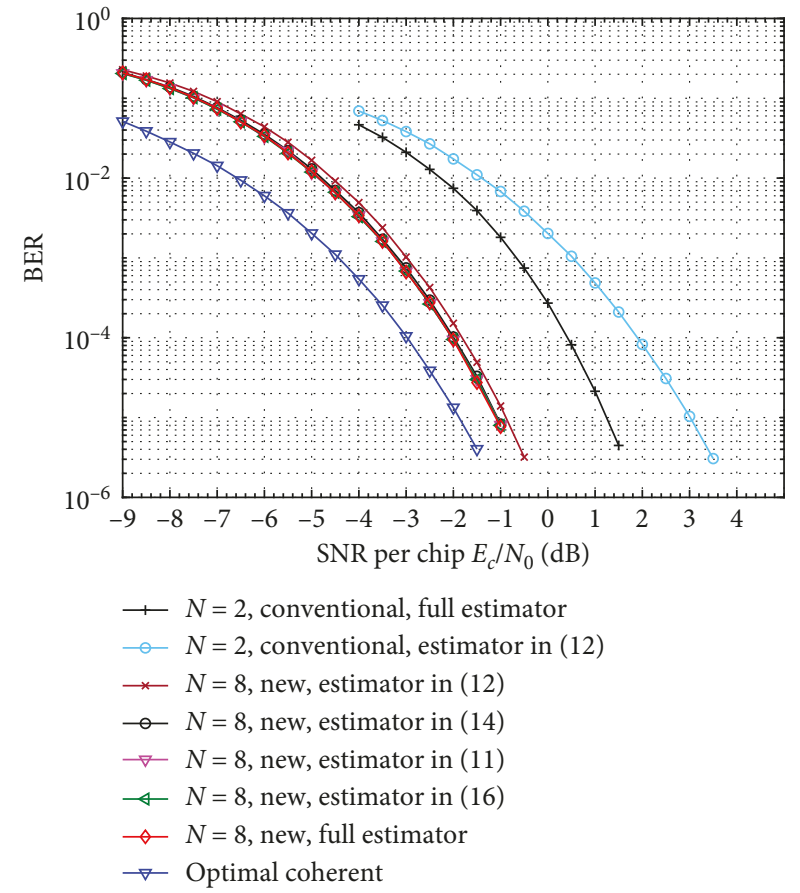

(a)

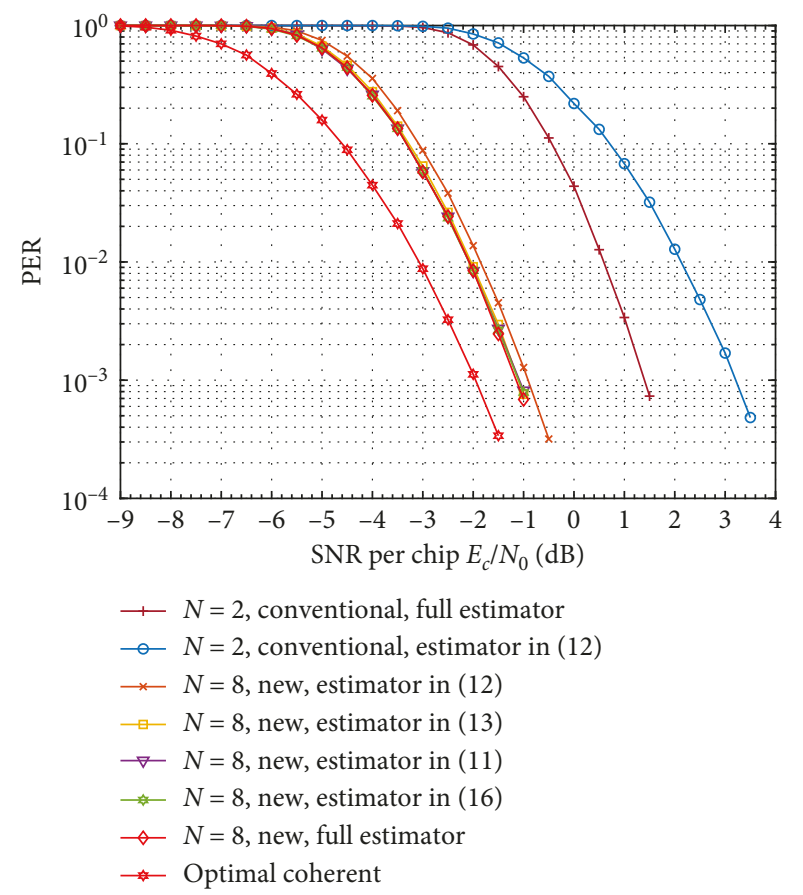

(b)

FIGURE 12: Detection performance comparison for two detection strategies over pure AWGN channel. (a) BER performance and (b) PER performance.

TABLE 3: Supply current for transmission when PER $=1 \times 10^{-3}$ with the MSDD in the sink node over the normalized slow Rayleigh fading channel, and $N=6$.

\begin{tabular}{lcc}
\hline Observation window length $N$ & SNR gain $(\mathrm{dB})$ & Supply current $I(\mathrm{~mA})$ \\
\hline 3 & 1.20 & 24.7 \\
4 & 1.80 & 23.2 \\
5 & 2.00 & 23.0 \\
6 & 2.10 & 22.5 \\
\hline
\end{tabular}

TABLE 4: Transmission energy gain achieved by the new MSDD in the sink node over the normalized slow Rayleigh fading channel, and $N=6$.

\begin{tabular}{lccc}
\hline Observation window length $N$ & Consumed energy $(\mu J)$ & Gain $(\mu J)$ & Energy saving $(\%)$ \\
\hline 3 & 360.0 & 12.96 & 3.5 \\
4 & 338.4 & 34.56 & 9.3 \\
5 & 331.2 & 41.76 & 11.2 \\
6 & 324.0 & 48.96 & 13.1 \\
\hline
\end{tabular}

6.6. Robustness of the MSDD Scheme to CFO over Pure AWGN Channel. In Figures 14 to 17 , we set the observation window length $N$ from 3 and 6 and give the corresponding BER and PER performance versus the CFO. In Figures 14 to 17, the black line denotes the detection scheme with the estimator where $\tan ^{-1}(x) \approx x$ is involved, and the red line represents the detection scheme with the proposed estimator where both $\sin ^{-1}(x) \approx x$ and $|Y| \approx 1$ are involved. For the convenience of comparison, the green line is the detection scheme with the estimator where only $\sin ^{-1}(x) \approx x$ is involved. Clearly, the robustness of our new MSDD scheme to
CFO is much stronger than that of the conventional scheme as indicated in Figures 14 to 17. Also, the proposed algorithm achieves much better performance, which is nearuniform across a frequency range of $-80 \mathrm{ppm}$ and $80 \mathrm{ppm}$ at all SNRs for all observation window lengths in the simulations. Hence, our proposed MSDD with any estimator is CFO insensitive.

6.7. Robustness of the New MSDD Scheme to CPO over Pure AWGN Channel. As in [12], the detection behavior of the 


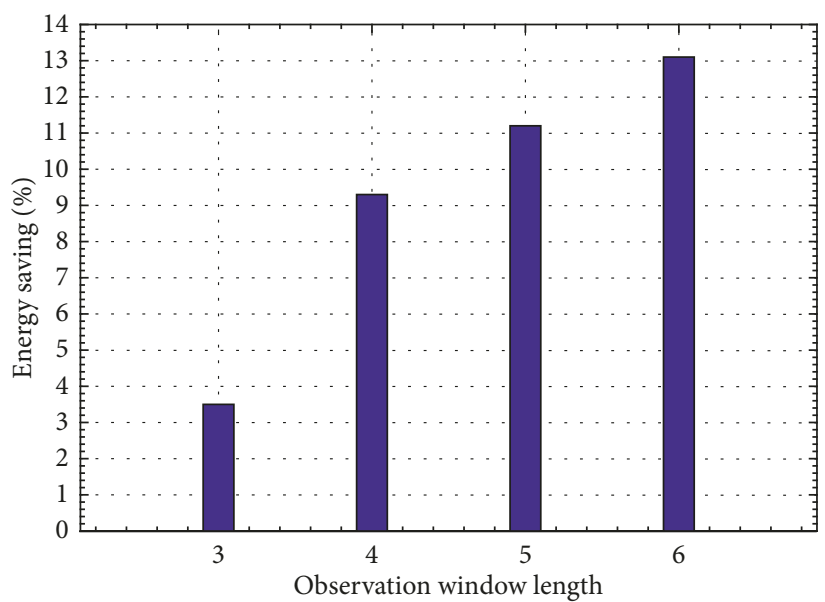

FIGURE 13: Transmission energy saving achieved by the MSDD in the sink node over the normalized slow Rayleigh fading channel.

TABLE 5: Supply current for transmission when PER $=1 \times 10^{-3}$ with the MSDD in the sink node over pure AWGN channel, and $N=6$.

\begin{tabular}{lcc}
\hline Observation window length $N$ & SNR gain $(\mathrm{dB})$ & Supply current $I$ (mA) \\
\hline 3 & 1.15 & 25.0 \\
4 & 1.70 & 23.5 \\
5 & 2.00 & 23.0 \\
6 & 2.10 & 22.5 \\
\hline
\end{tabular}

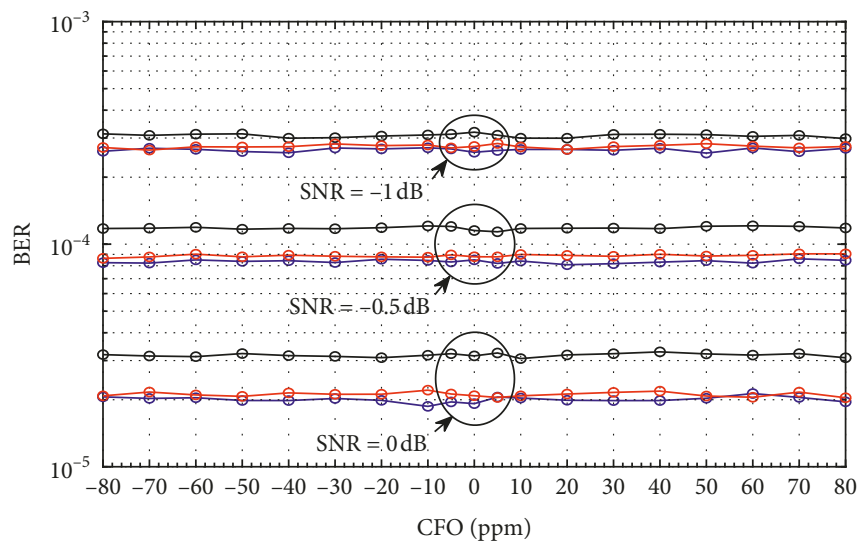

(a)

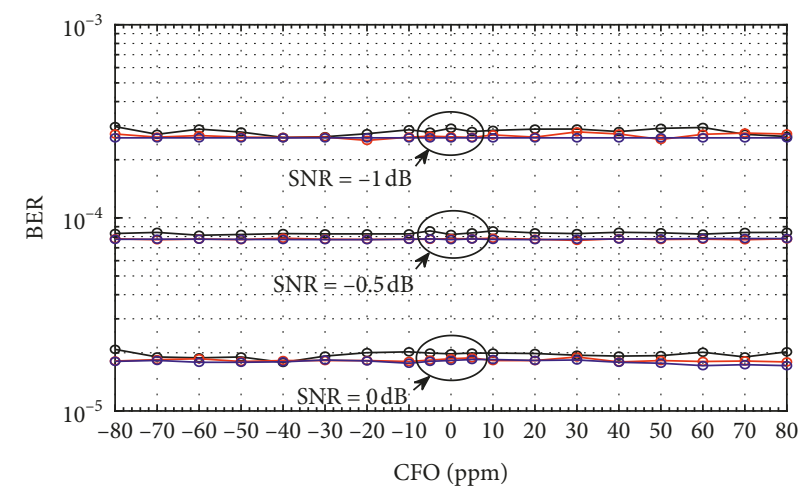

(c)

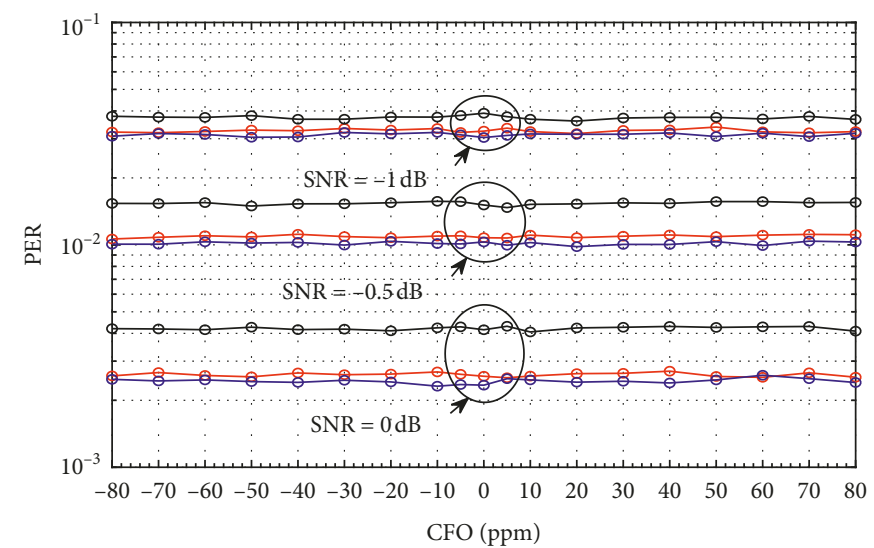

(b)

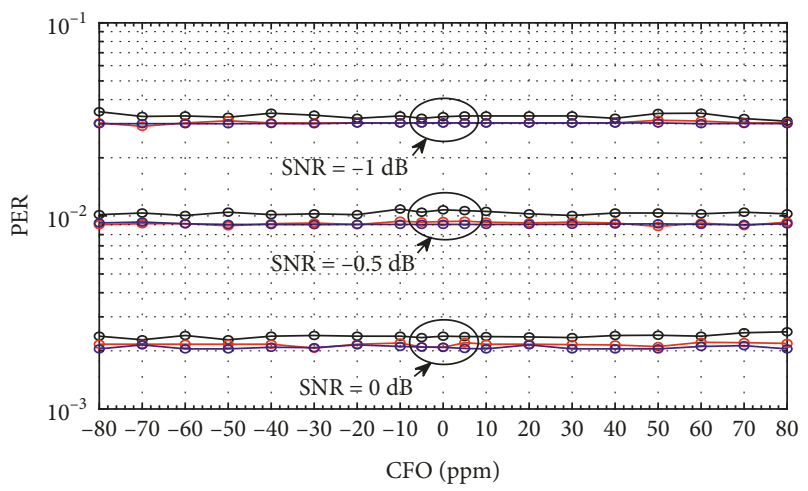

(d)

FIGURE 14: Detection performance versus CFO over pure AWGN channel for $N=3$. (a) BER of the conventional detection strategy; (b) PER of the conventional detection strategy; (c) BER of the new detection strategy; (d) PER of the new detection strategy. 


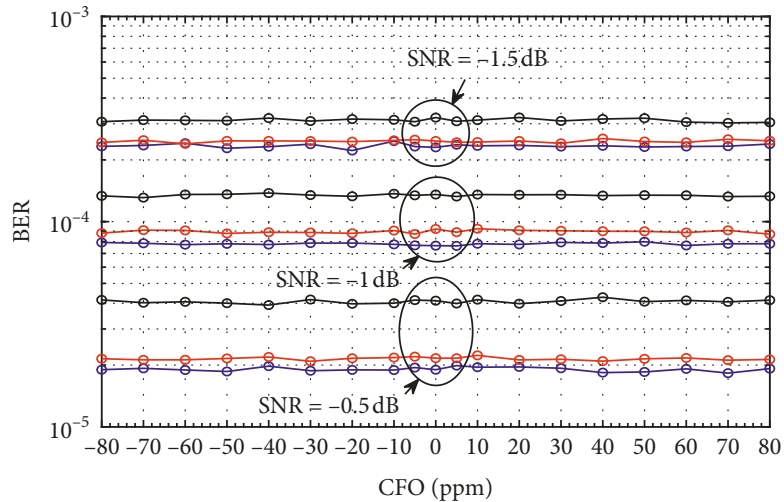

(a)

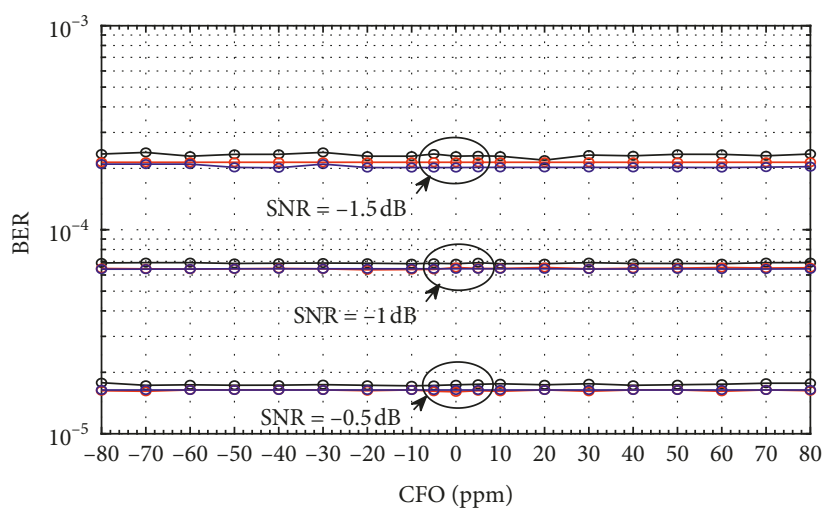

(c)

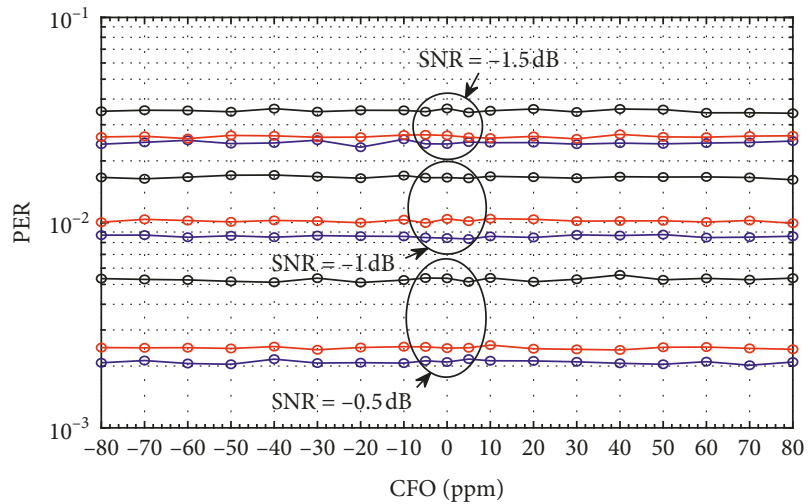

(b)

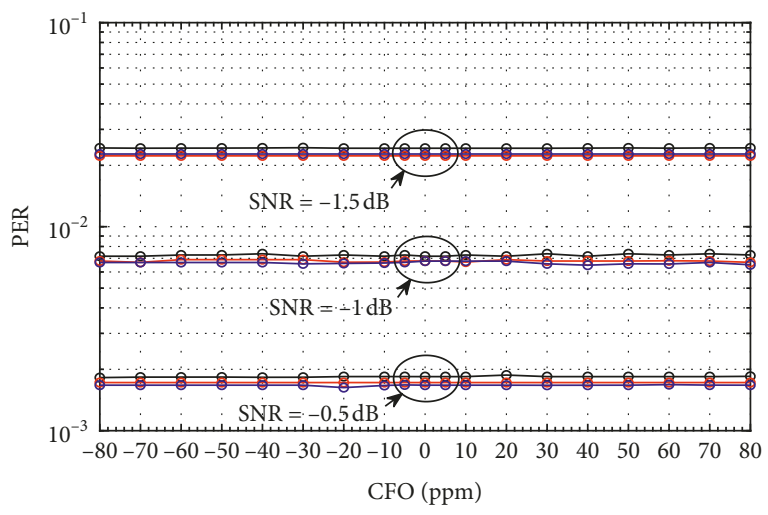

(d)

FIGURE 15: Detection performance versus CFO over pure AWGN channel, $\theta$ is uniform distribution in $(-\pi, \pi)$, and $N=4$. (a) BER of the conventional detection strategy; (b) PER of the conventional detection strategy; (c) BER of the new detection strategy; (d) PER of the new detection strategy.

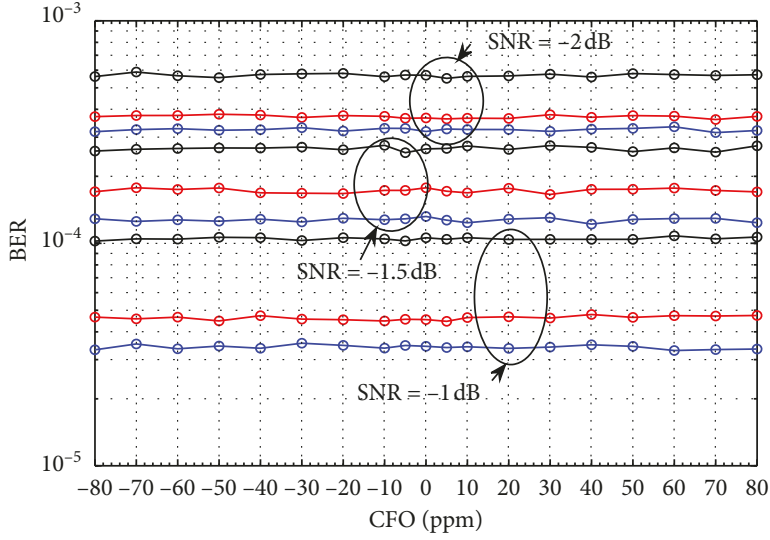

(a)

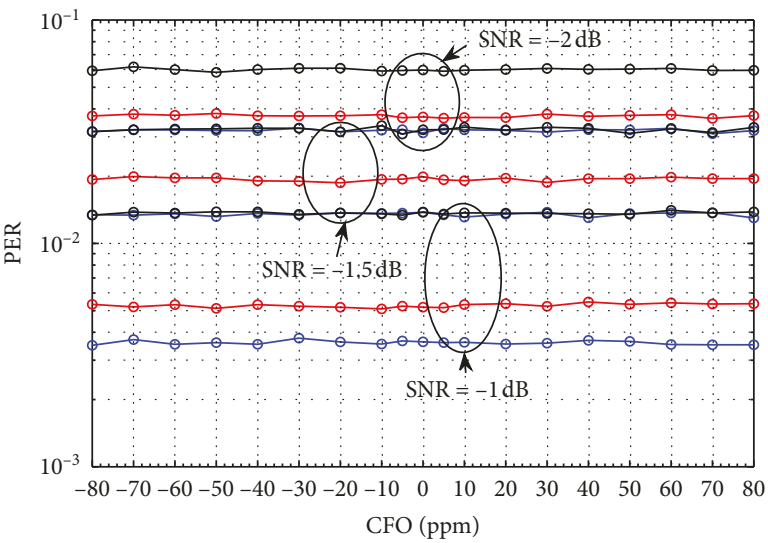

(b)

Figure 16: Continued. 


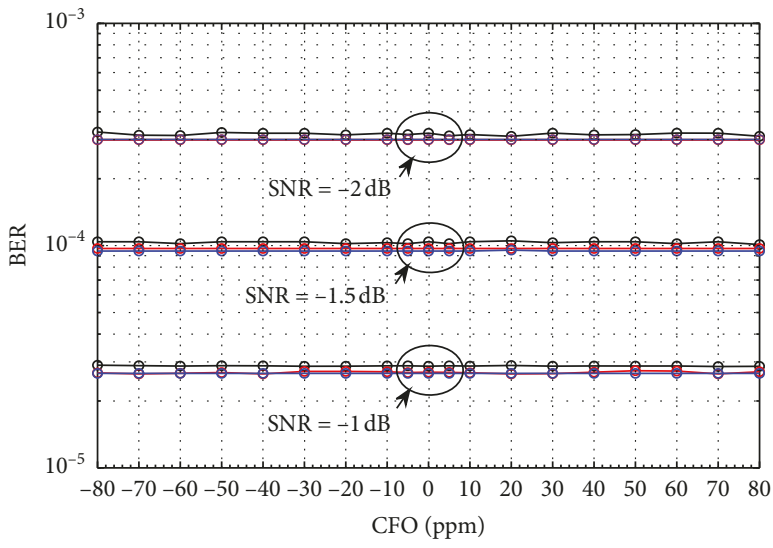

(c)

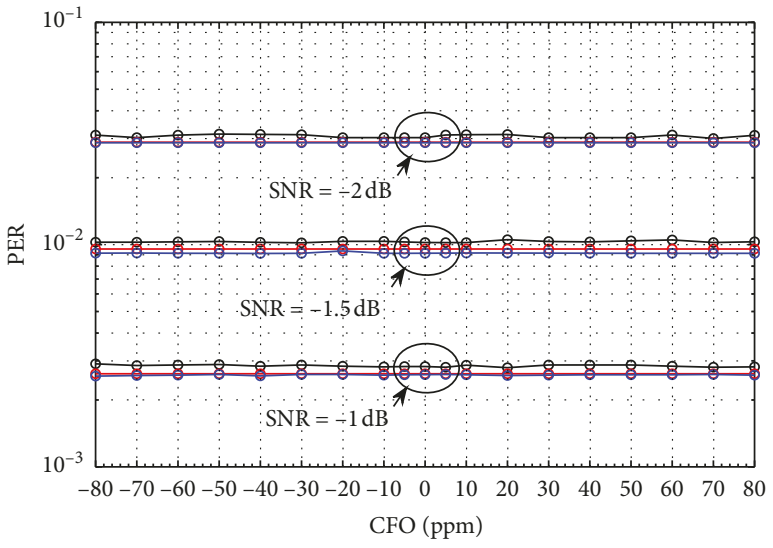

(d)

FIGURE 16: Detection performance versus CFO over pure AWGN channel, $\theta$ is uniform distribution in $(-\pi, \pi)$, and $N=5$. (a) BER of the conventional detection strategy; (b) PER of the conventional detection strategy; (c) BER of the new detection strategy; (d) PER of new the detection strategy.

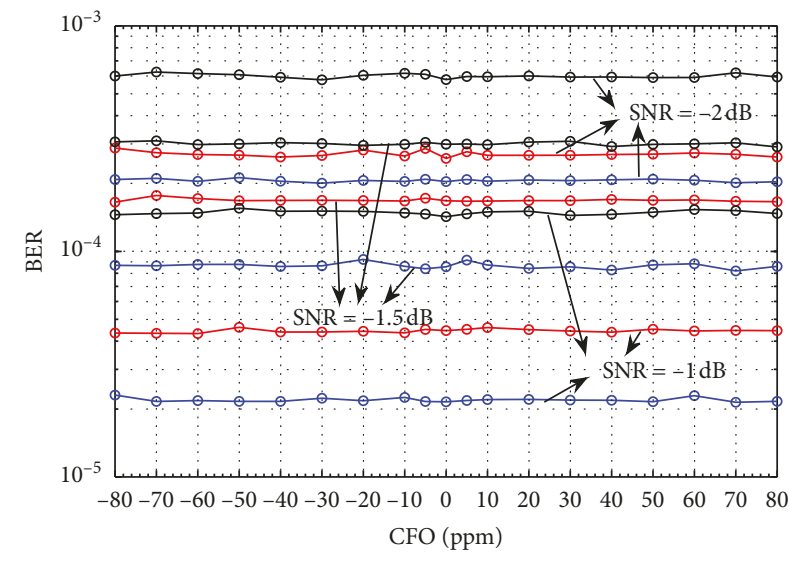

(a)

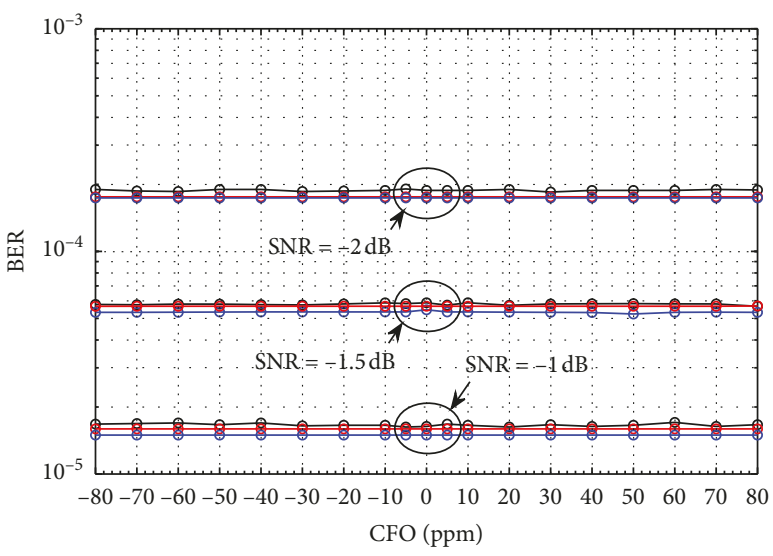

(c)

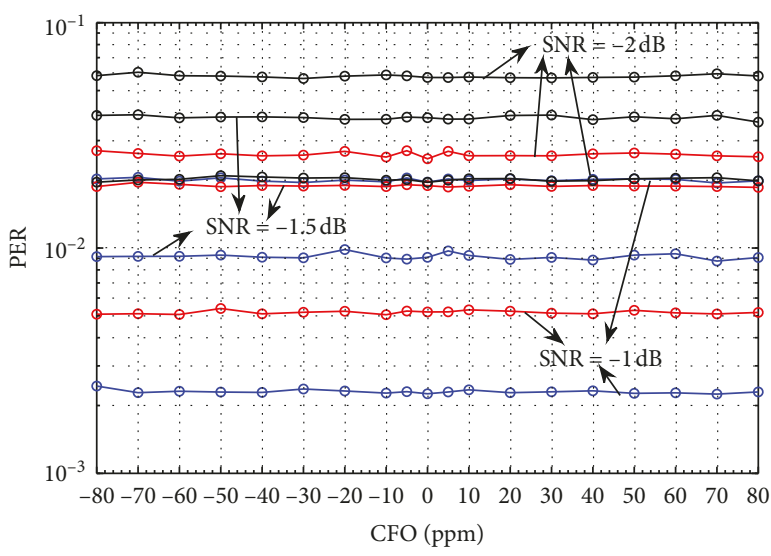

(b)

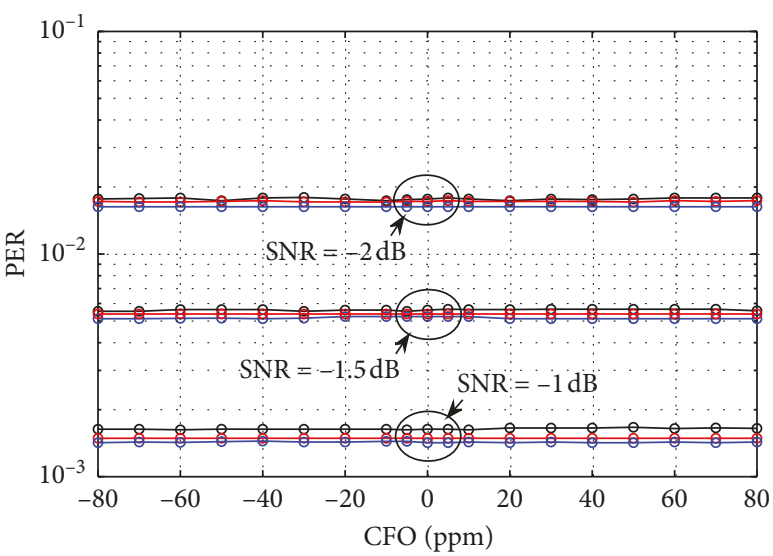

(d)

FIGURE 17: Detection performance versus CFO over pure AWGN channel, $\theta$ is uniform distribution in $(-\pi, \pi)$, and $N=6$. (a) BER of the conventional detection strategy; (b) PER of the conventional detection strategy; (c) BER of the new detection strategy; (d) PER of the new detection strategy.

proposed MSDD scheme with changing carrier phase has also been investigated in Figures 18 to 20, wherein $N$ is set to be 3. The reader can refer to [12] for a detailed discussion about the simulation parameters. As observed from Figures 18 to 20, the new MSDD scheme is robust to dynamic phase jitter. With larger observation window lengths, the 


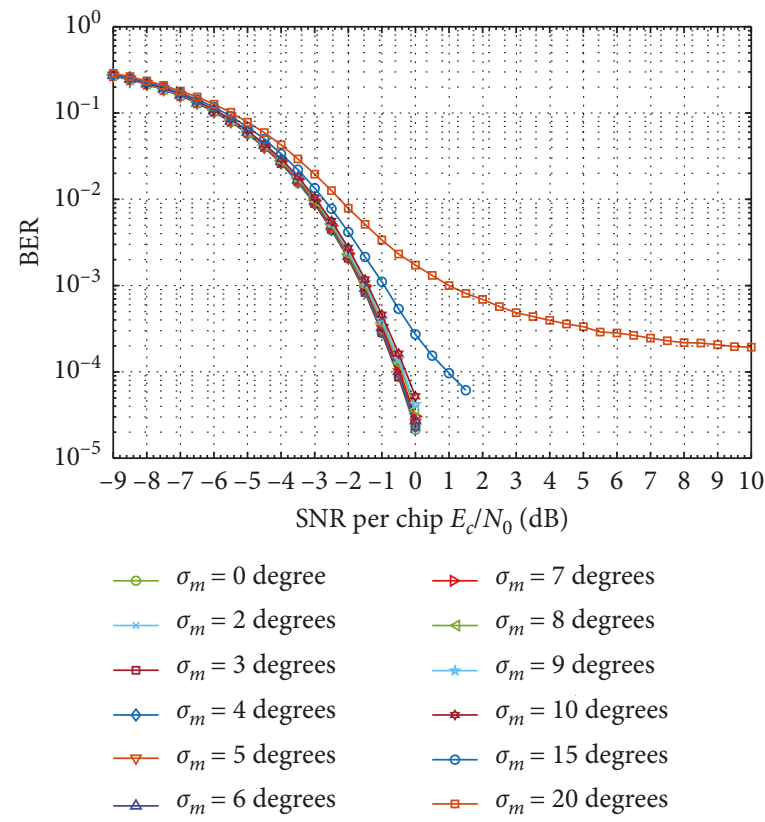

(a)

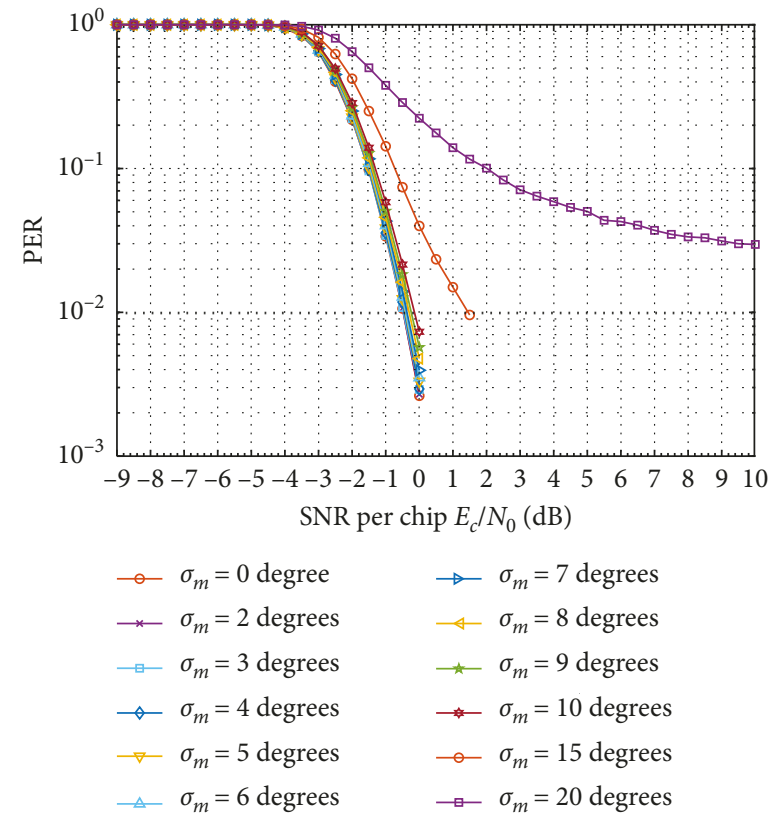

(b)

FIGURE 18: Detection performance of the new MSDD with (11) over pure AWGN channel with dynamic CPO, $\theta$ is a Wiener process, and $N=3$. (a) BER performance and (b) PER performance.

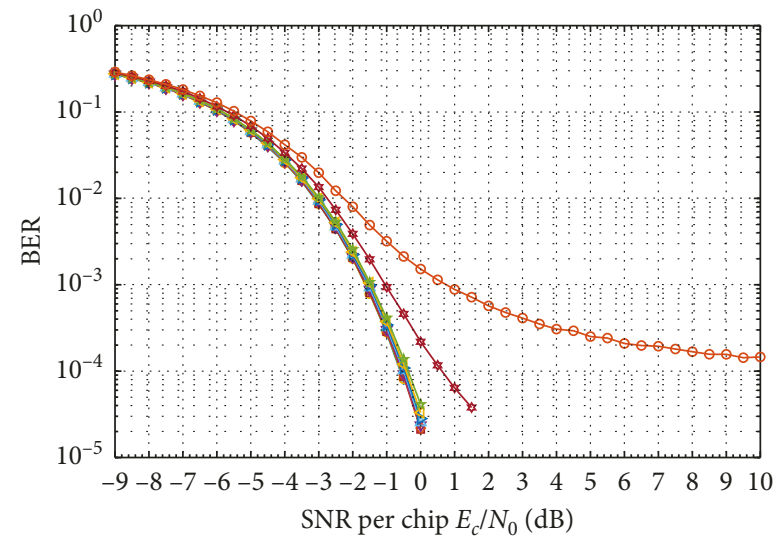

$$
\begin{aligned}
& \leftarrow \sigma_{m}=0 \text { degree } \triangle-\sigma_{m}=7 \text { degrees } \\
& \multimap \sigma_{m}=2 \text { degrees } \rightarrow \sigma_{m}=8 \text { degrees } \\
& \text { * } \sigma_{m}=3 \text { degrees } \triangleleft \sigma_{m}=9 \text { degrees } \\
& \square \sigma_{m}=4 \text { degrees } \quad \text { * } \sigma_{m}=10 \text { degrees } \\
& \rightarrow \sigma_{m}=5 \text { degrees } \quad * \sigma_{m}=15 \text { degrees } \\
& \rightarrow \sigma_{m}=6 \text { degrees }
\end{aligned}
$$

(a)

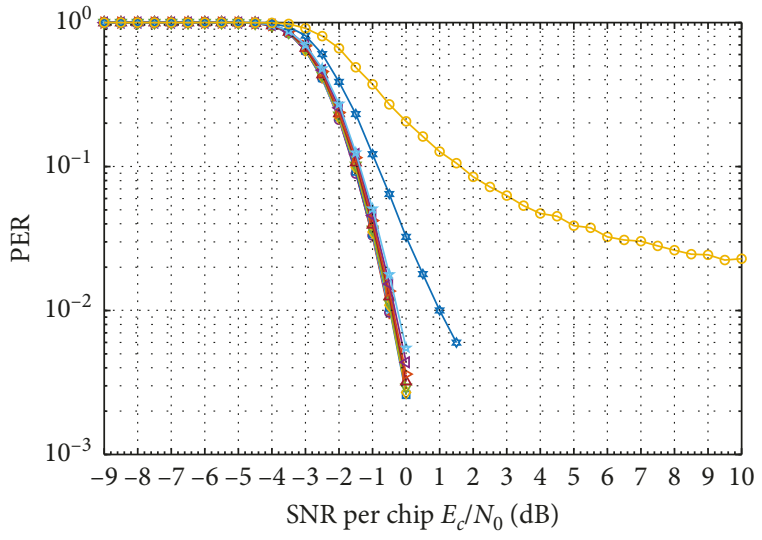

$$
\begin{aligned}
& \because \sigma_{m}=0 \text { degree } \quad \triangle \sigma_{m}=7 \text { degrees } \\
& \rightarrow \sigma_{m}=2 \text { degrees } \rightarrow \sigma_{m}=8 \text { degrees } \\
& \text { * } \sigma_{m}=3 \text { degrees } \varangle \sigma_{m}=9 \text { degrees } \\
& \rightarrow \sigma_{m}=4 \text { degrees } \quad \text { औ } \sigma_{m}=10 \text { degrees } \\
& \because \sigma_{m}=5 \text { degrees } \quad \because \sigma_{m}=15 \text { degrees } \\
& \rightarrow \sigma_{m}=6 \text { degrees }
\end{aligned}
$$

(b)

FIGURE 19: Detection performance of the new MSDD with (20) over pure AWGN channel with dynamic CPO, $\theta$ is a Wiener process, and $N=3$. (a) BER performance and (b) PER performance.

performance under dynamic channel was also developed, which however is not given here.

6.8. Computational Complexity. We assume that the same estimator is equipped with the detection scheme. Thus, the first complexity difference is determined by the measurement in (14). Moreover, the other complexity difference is determined by the compensation process. In Tables 6 to 9 , the complexity is compared over pure AWGN channel. Note that, we also consider the maximum values for $L_{1}, L_{2}$, and $L_{3}$, i.e., $L_{1}=32$ and $L_{2}=L_{3}=15$. Block diagram of complex 


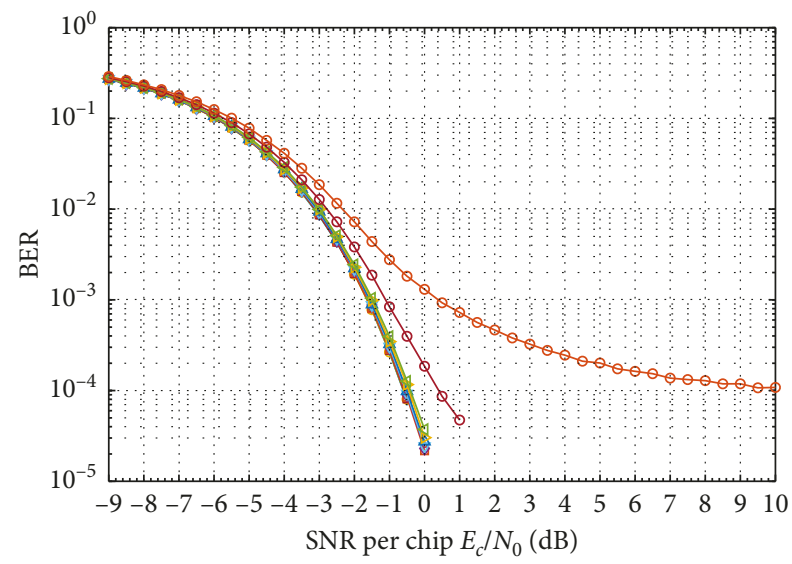

$$
\begin{aligned}
& \longrightarrow \sigma_{m}=0 \text { degree } \quad \nabla \sigma_{m}=7 \text { degrees } \\
& \therefore \sigma_{m}=2 \text { degrees } \quad \triangle \sigma_{m}=8 \text { degrees } \\
& \star \sigma_{m}=3 \text { degrees } \\
& \rightarrow \sigma_{m}=4 \text { degrees } \\
& \rightarrow \sigma_{m}=5 \text { degrees } \\
& \diamond \sigma_{m}=6 \text { degrees } \\
& \gg \sigma_{m}=9 \text { degrees } \\
& \longleftarrow \sigma_{m}=10 \text { degrees } \\
& \multimap \sigma_{m}=15 \text { degrees } \\
& \circ \sigma_{m}=20 \text { degrees }
\end{aligned}
$$

(a)

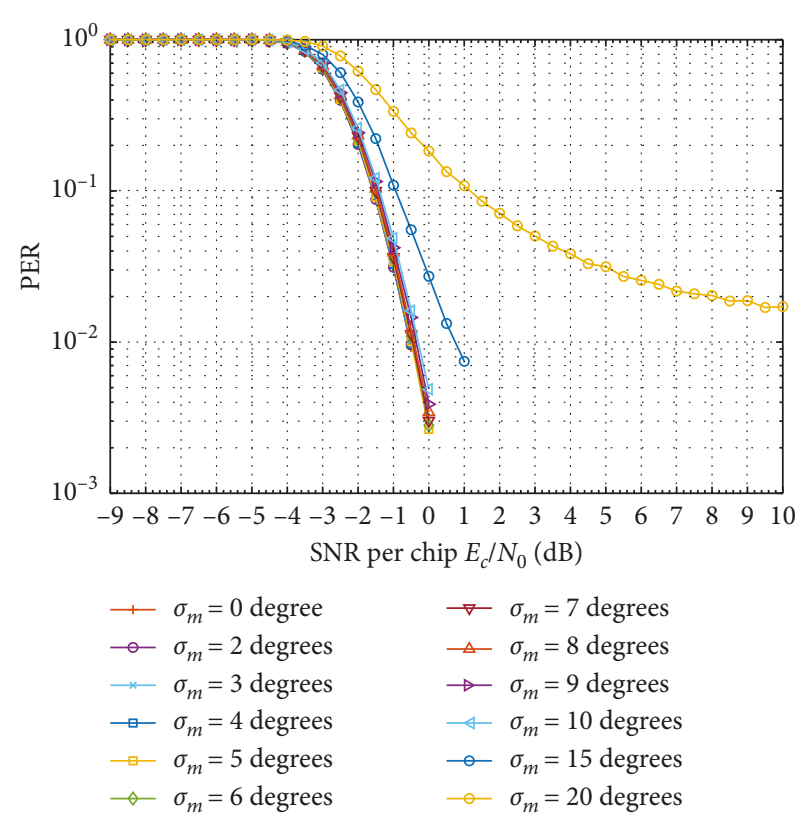

(b)

FIGURE 20: Detection performance of the new MSDD with (10) over pure AWGN channel with dynamic CPO, $\theta$ is a Wiener process, and $N=3$. (a) BER performance and (b) PER performance.

TABLE 6: Implementation complexity of the measurement given in equation (2) of [15] for conventional MSDD over pure AWGN channel.

\begin{tabular}{lcc}
\hline $\begin{array}{l}\text { Observation window } \\
\text { length } N\end{array}$ & $\begin{array}{c}\text { Complex } \\
\text { multiplication }\end{array}$ & $\begin{array}{c}\text { Complex } \\
\text { addition }\end{array}$ \\
\hline 2 & 465 & 465 \\
3 & 465 & 465 \\
4 & 465 & 465 \\
5 & 465 & 465 \\
6 & 465 & 465 \\
\hline
\end{tabular}

TABLE 7: Complexity analysis of the compensation process in equation (2) of [15] for conventional MSDD over pure AWGN channel.

\begin{tabular}{lc}
\hline Observation window length $N$ & Complex multiplication \\
\hline 2 & 30 \\
3 & 45 \\
4 & 60 \\
5 & 75 \\
6 & 80 \\
\hline
\end{tabular}

multiplication is shown in Figure 3 of [22]. Complex addition means addition operation of two complex numbers. As shown in Tables 6 to 9, the implementation complexity of our proposed new strategy is not much higher than that of the conventional scheme. For example, for $N=3$, additional 408 complex multiplications and 450 complex additions are required for the new strategy. As $N$ increases to 6, these two numbers increase to 1645 and 1710 , respectively.
TABLE 8: Implementation complexity of the measurement given in (5) for the proposed MSDD over pure AWGN channel.

\begin{tabular}{lcc}
\hline Observation window length $N$ & $\begin{array}{c}\text { Complex } \\
\text { multiplication }\end{array}$ & $\begin{array}{c}\text { Complex } \\
\text { addition }\end{array}$ \\
\hline 2 & 465 & 465 \\
3 & 915 & 915 \\
4 & 1350 & 1350 \\
5 & 1770 & 1770 \\
6 & 2175 & 2175 \\
\hline
\end{tabular}

TABLE 9: Implementation complexity of the compensation process in (3) for the proposed MSDD over pure AWGN channel.

\begin{tabular}{lc}
\hline Observation window length $N$ & Complex multiplication \\
\hline 2 & 1 \\
3 & 3 \\
4 & 6 \\
5 & 10 \\
6 & 15 \\
\hline
\end{tabular}

\section{Conclusions and Future Work}

To eliminate the error floor of the conventional MSDD scheme for BPSK receivers in IEEE 802.15.4 WSNs, we have presented a new and robust detection strategy. The basic principle is that we wipe out the effect of the CFO after the bit-level sample autocorrelation operation. Much performance gain from differential and spread spectrum coding implemented in IEEE 802.15.4 BPSK physical layer is extracted, and much transmitting energy is saved no matter 
which estimator is configured. This makes it more attractive for WSNs applications, which are delay-tolerant but performance-sensitive.

Our proposed MSDD scheme can also be applied under fading channel in the presence of rapidly changing carrier phase and carrier frequency, although only a slow fading channel is considered. In this context, the non-data-aided detection method is preferred. All of these follow from the fact that the modulated data within the sample chip can be easily wiped out with the aid of any PN code using the crosscorrelation operation. We pay all our attention towards noncoherent detection for single-carrier modulation in WSNs. Orthogonal frequency division multiplexing (OFDM) has also been suggested to support higher data transmission rate $[33,34]$. Clearly, our proposed scheme cannot be directly used in multicarrier modulation communication system. This will be one of our future research directions.

\section{Data Availability}

All of the underlying data in our manuscript are available from the corresponding author upon request.

\section{Conflicts of Interest}

The authors declare no conflicts of interest.

\section{Acknowledgments}

This work was partially supported by the National Natural Science Foundation of China (project nos. 61701172, 41605122, 61701059, 61701062, 61801170, 61801171, and 61772175), Open Foundation of the Key Laboratory of Middle Atmosphere and Global Environment Observation, Institute of Atmospheric Physics, the Natural Science Foundation of Henan Province (project nos. 162300410097 and 162300410096), the Scientific and Technological Innovation Team of Colleges and Universities in Henan Province (project no. 20IRTSHN018), Chinese Academy of Sciences, Postdoctoral Science Foundation of University of Electronic Science and Technology of China (project no. Y02006023601721), and the Program for Science and Technology Innovation Talents in the University of Henan Province (Educational Committee) (project no. 17HASTIT025).

\section{References}

[1] J. Zhao, C. Qiao, R. S. Sudhaakar, and S. Yoon, "Improve efficiency and reliability in single-hop WSNs with transmitonly nodes," IEEE Transactions on Parallel and Distributed Systems, vol. 24, no. 3, pp. 520-534, 2003.

[2] L. Galluccio, G. Morabito, and S. Palazzo, "TC-Aloha: TCaloha: a novel access scheme for wireless networks with transmit-only nodes," IEEE Transactions on Wireless Communications, vol. 12, no. 8, pp. 3696-3709, 2013.

[3] B. Cao, L. Zhang, Y. Li, D. Feng, and W. Cao, "Intelligent offloading in multi-access edge computing: a state-of-the-art review and framework," IEEE Communications Magazine, vol. 57, no. 3, pp. 56-62, 2019.
[4] B. Cao, S. Xia, J. Han, and Y. Li, "A distributed game methodology for crowdsensing in uncertain wireless scenario," IEEE Transactions on Mobile Computing, p. 1, 2019.

[5] B. F. Ji, Z. Z. Chen, S. D. Chen, B. Zhou, C. Li, and W. Hong, "Joint optimization for ambient backscatter communication system with energy harvesting for IoT," Mechanical Systems and Signal Processing, vol. 135, Article ID 106412, 2019.

[6] B. Radunovic, H. L. Truong, and M. Weisenhorn, "Receiver architectures for UWB-Based transmit-only sensor networks," in Proceedings of the IEEE International Conference on Ultrawideband, pp. 379-384, Zurich, Switzerland, September 2005.

[7] B. Blaszczyszyn and B. Radunovic, "Using transmit-only sensors to reduce deployment cost of wireless sensor networks," in Proceedings of the 2008 Proceedings IEEE INFOCOM-The 27th Conference on Computer Communications, pp. 1202-1210, Phoenix, AZ, USA, April 2008.

[8] IEEE 802.15.4-2011 Standard, IEEE Standard for Local and Metropolitan Area Network-Part 15.4: Low Rate Wireless Personal Area Networks (LR-WPANs), IEEE Press, Piscataway, NJ, USA, 2011.

[9] H. W. Tseng, A. C. Pang, J. H. Chen, and C. F. Kuo, "An adaptive contention control strategy for IEEE 802.15.4-based wireless sensor networks," IEEE Transactions on Vehicular Technology, vol. 58, no. 9, pp. 5164-5173, 2009.

[10] H. J. Jeon, T. Demeechai, W. G. Lee, and D. H. Kim, "IEEE 802.15.4 BPSK receiver architecture based on a new efficient detection scheme," IEEE Transactions on Signal Processing, vol. 58, no. 9, pp. 4711-4719, 2010.

[11] S. Lee, H. Kwon, Y. Jung, and J. Kim, "Efficient non-coherent demodulation scheme for IEEE 802.15.4 LR-WPAN systems," Electronics Letters, vol. 43, no. 16, pp. 879-880, 2007.

[12] G. Zhang, H. Wen, L. Wang, L. Song, J. Tang, and R. Liao, "Simple and robust near-optimal single differential detection scheme for IEEE 802.15.4 BPSK receivers," IET Communications, vol. 13, no. 2, pp. 186-197, 2019.

[13] D. Divsalar and M. K. Simon, "Multiple-symbol differential detection of MPSK," IEEE Transactions on Communications, vol. 38, no. 3, pp. 300-308, 1990.

[14] D. Makrakis and K. Feher, "Optimal noncoherent detection of PSK signals," Electronics Letters, vol. 26, no. 6, pp. 398-400, 1990.

[15] G. Zhang, H. Wen, L. Wang et al., "Multiple symbol differential detection scheme for IEEE 802.15.4 BPSK receivers," IEICE Transactions on Fundamentals of Electronics, Communications and Computer Sciences, vol. E101-A, no. 11, pp. 1975-1979, 2018.

[16] L. Li, R. G. Maunder, B. M. Al-Hashimi, and L. Hanzo, “An energy-efficient error correction scheme for IEEE 802.15.4 wireless sensor networks," IEEE Transactions on Circuits and Systems II: Express Briefs, vol. 53, no. 3, pp. 233-237, 2010.

[17] Y. Li, J. Liu, B. Cao, and C. Wang, "Joint optimization of radio and virtual machine resources with uncertain user demands in mobile cloud computing," IEEE Transactions on Multimedia, vol. 20, no. 9, pp. 2427-2438, 2018.

[18] Y. Li, S. Xia, M. Y. Zheng, B. Cao, and Q. Liu, "Lyapunov optimization based trade-off policy for mobile cloud offloading in heterogeneous wireless networks," IIEEE Transactions on Cloud Computing, 2019.

[19] Y. Li, Y. J. Liang, Q. L. Liu, and H. G. Wang, "Resources allocation in multi-cell D2D Communications for internet of Things," IEEE Internet of Things Journal, vol. 2, no. 6, pp. 504-514, 2018.

[20] F. Barac, M. Gidlund, and T. T. Zhang, "CLAP: Chip-level augmentation of IEEE 802.15.4 PHY for error-intolerant 
WSN communication," in Proceedings of the International Conference on Vehicular Technology, pp. 1-7, Glasgow, UK, May 2015.

[21] S. Park, D. Park, H. Park, and K. Lee, "Low-complexity frequency-offset insensitive detection for orthogonal modulation,” Electronics Letters, vol. 41, no. 22, pp. 1226-1227, 2005.

[22] D. Park, C. S. Park, and K. Lee, "Simple design of detector in the presence of frequency offset for IEEE 802.15.4 LRWPANs," IEEE Transactions on Circuits and Systems II: Express Briefs, vol. 56, no. 4, pp. 330-334, 2009.

[23] C. Wang, Y. P. Liu, R. Luo, and H. Z. Yang, "A low-complexity symbol-level differential detection scheme for IEEE 802.15.4 O-QPSK signals," in Proceedings of the International Conference on Wireless Communications \& Signal Processing, pp. 1-6, Huangshan, China, October 2013.

[24] J.-H. Do, J. S. Han, H. J. Choi, U. Lee, and S.-H. Min, "A coherent detection-based symbol detector algorithm for 2.45 GHz LR-WPAN receiver," in Proceedings of the TENCON 2005-2005 IEEE Region 10 Conference, vol. 10, pp. 1-6, Melbourne, Qld., Australia, November 2005.

[25] A. Willig, K. Matheus, and A. Wolisz, "Wireless technology in industrial networks," Proceedings of the IEEE, vol. 93, no. 6, pp. 1130-1151, 2005.

[26] R. G. Maunder, A. S. Weddell, G. V. Merrett, B. M. AlHashimi, and L. Hanzo, "Iterative decoding for redistributing energy consumption in wireless sensor networks," in Proceedings of the 17th International Conference on Computer Communications and Networks, pp. 1-6, St. Thomas, US Virgin Islands, USA, August 2008.

[27] Low Power, 700/800/900 MHz Transceiver for ZigBee, IEEE 802.15.4, 6LoWPAN, and ISM Applications Datasheet, 2015.

[28] D. Divsalar and M. K. Simon, "Maximum-likelihood differential detection of uncoded and trellis coded amplitude phase modulation over AWGN and fading channels-metrics and performance," IEEE Transactions on Communications, vol. 42, no. 1, pp. 76-89, 1994.

[29] M. K. Simon and D. Divsalar, "Multiple symbol partially coherent detection of MPSK," IEEE Transactions on Communications, vol. 4, no. 234, pp. 652-657, 1994.

[30] V. Lottici and Z. Tian, "Multiple symbol differential detection for UWB communications," IEEE Transactions on Wireless Communications, vol. 7, no. 5, pp. 1656-1666, 2008.

[31] G. Colavolpe and R. Raheli, "Noncoherent sequence detection," IEEE Transactions on Communications, vol. 47, no. 9, pp. 1376-1385, 1999.

[32] P. Soderquist and M. Leeser, "Area and performance tradeoffs in floating-point divide and square-root implementations," ACM Computing Surveys, vol. 28, no. 3, pp. 518-564, 1996.

[33] IEEE 802.15.4-2015 Standard, IEEE Standard for Low-Rate Wireless Networks, IEEE Press, Piscataway, NJ, USA, 2015.

[34] M. Wen, E. Basar, Q. Li, B. X. Zheng, and M. Zhang, "Multiple-mode orthogonal frequency division multiplexing with index modulation," IEEE Transactions on Communications, vol. 65, no. 9, pp. 3892-3906, 2013. 


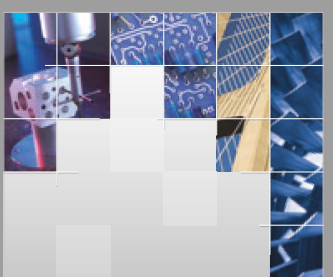

\section{Enfincering}
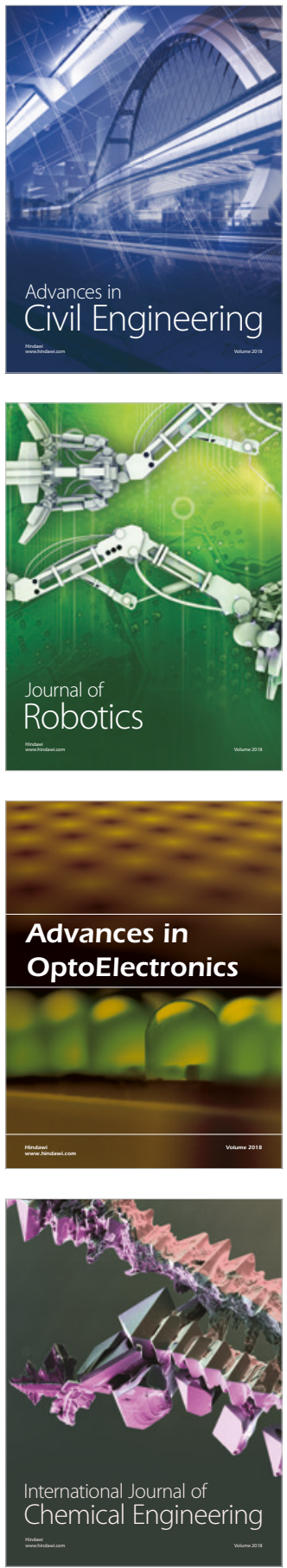

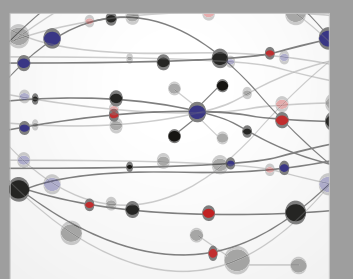

\section{Rotating \\ Machinery}

The Scientific World Journal

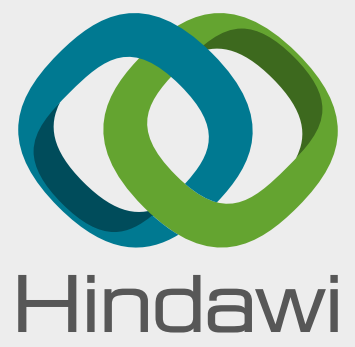

Submit your manuscripts at

www.hindawi.com
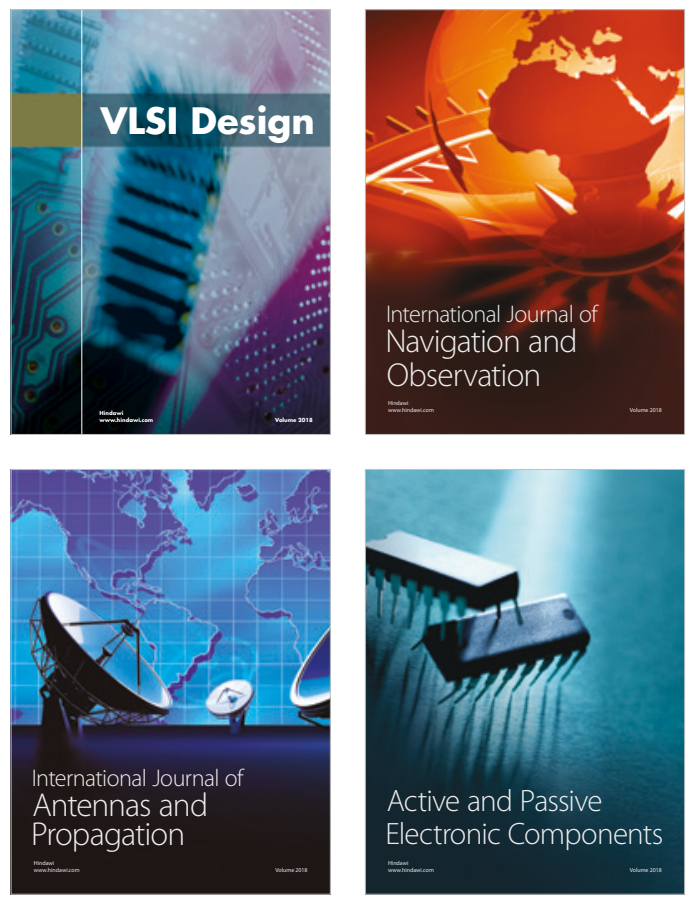
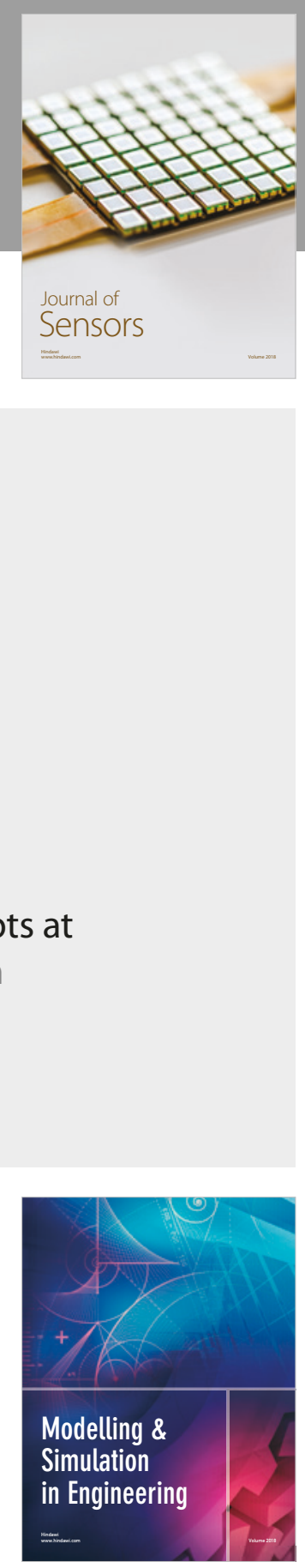

\section{Advances \\ Multimedia}
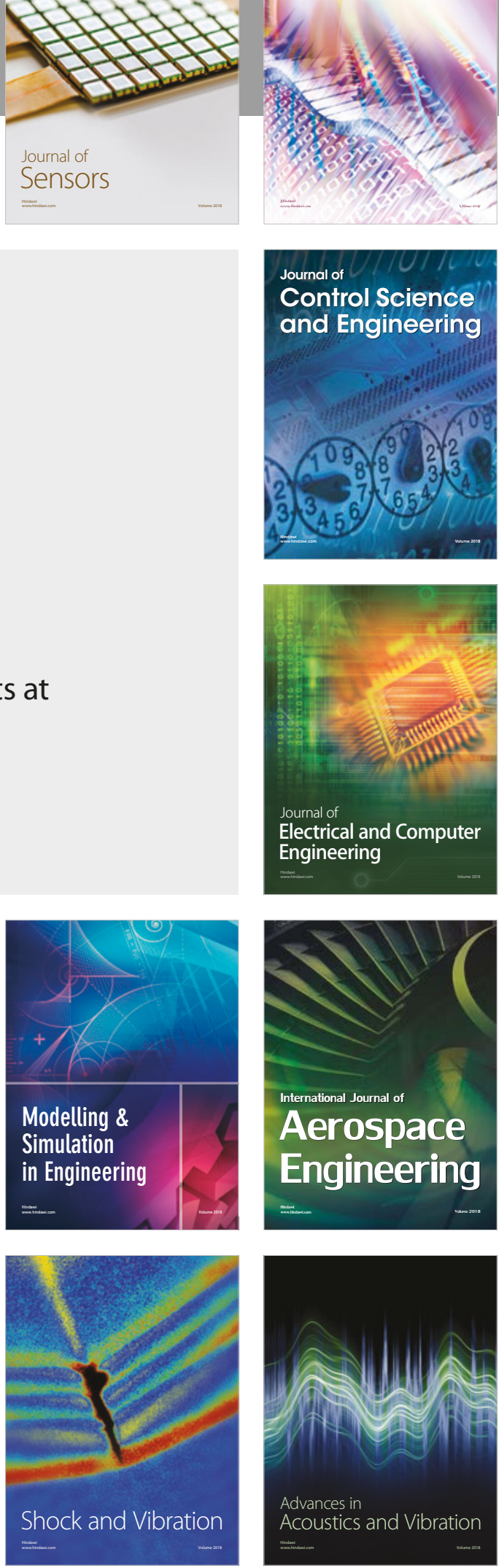